\title{
Gabriela Dinescu
}

Weak spectral equivalence and weak spectral convergence

Czechoslovak Mathematical Journal, Vol. 34 (1984), No. 2, 203-226

Persistent URL: http://dml.cz/dmlcz/101944

\section{Terms of use:}

(C) Institute of Mathematics AS CR, 1984

Institute of Mathematics of the Czech Academy of Sciences provides access to digitized documents strictly for personal use. Each copy of any part of this document must contain these Terms of use.

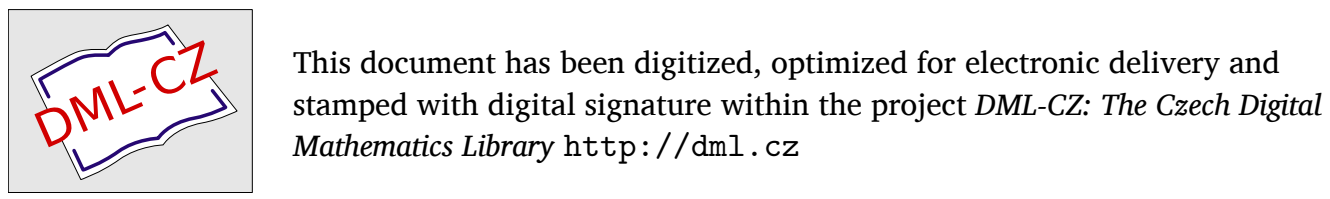




\title{
WEAK SPECTRAL EQUIVALENCE AND WEAK SPECTRAL CONVERGENCE
}

\author{
Gabriela Dinescu, Bucharest
}

(Received August 3, 1982)

In this paper we define the weak spectral pseudo-distance between two operators in $\mathscr{L}(X)(X$ being a Banach space), starting from a formula of $\mathrm{F}$. $\mathrm{H}$. Vasilescu $([8])$. Using this pseudo-distance, we introduce the notions of weak spectral equivalence and weak spectral convergence. We show (Theorem 2.21) that the weak spectral equivalence is really weaker than the usual spectral equivalence.

In this framework we prove some familiar results concerning the spectral equivalence as: the equality of spectra and of the subspaces $X_{T}(F)$ (for definitions see Sec. 1), the permanence of the s.v.e.p. (cf. Definition 1.6) and of the decomposability (see Theorem 2.16) as well as the connections with functional calculus and with the similarity. On the other hand, we prove that the weak spectral convergence also preserves the s.v.e.p. and the decomposability (see Theorems 3.8 and 3.9).

In order to obtain conditions upon which the weak spectral equivalence is preserved by passing to subspaces and quotient spaces, properties of permanence of the local spectrum are studied. Related to this, the notion of the $\Omega$-analytically invariant subspace is introduced and the formulas for the local spectrum in some particular examples of quotient spaces are given.

\section{PRELIMINARIES}

Let us recall the definition of the Hausdorff distance between two sets and the basic definitions from the spectral theory of operators in a Banach space.

a) The Hausdorff distance between two sets. Definition 1.1. Let $A, B$ be two non-void subsets of $\mathbb{C}$. We define

$$
\begin{gathered}
\delta(A, B)=\sup _{\lambda \in A} \inf _{\mu \in B}|\lambda-\mu|, \\
p(A, B)=\max \{\delta(A, B) ; \delta(B, A)\} .
\end{gathered}
$$

The following properties are known or easy to prove:

Lemma 1.2.

a) $\delta(A, B)=0 \Leftrightarrow A \subset \bar{B}$ 
b) $\delta(A, B)=\delta(B, A)=0 \Leftrightarrow \bar{A}=\bar{B}$;

c) $\delta(A, B)=\delta(\bar{A}, \bar{B})$.

Lemma 1.3. For non-void sets $A, B, C$ in $\mathbb{C}$,

$$
\begin{gathered}
\delta(A, B) \leqq \delta(A, C)+\delta(C, B), \\
p(A, B) \leqq p(A, C)+p(C, B) .
\end{gathered}
$$

Lemma 1.4. Let $\left\{A_{\alpha}\right\}_{\alpha}$ and $\left\{B_{\beta}\right\}_{\beta}$ be two families of non-void sets in $\mathbb{C}$. We have

$$
\delta\left(\bigcup_{\alpha} A_{\alpha}, \bigcup_{\beta} B_{\beta}\right)=\sup _{\alpha} \inf _{\beta} \delta\left(A_{x}, B_{\beta}\right) .
$$

Remark 1.5. Adopting the conventions $\sup _{i \in \emptyset} \lambda_{i}=0$ and $\inf _{i \in \emptyset} \lambda_{i}=\infty$, where $\left\{\lambda_{i}\right\}$ is a family of positive (finite or not) numbers, Definition 1.1 and Lemmas 1.2, 1.3 and 1.4 include also the case when $A$ or $B$ (or both) are the void set. We obtain, for $A$ non-void,

$$
\delta(\emptyset, A)=0 ; \quad \delta(A, \emptyset)=\infty ; \quad \delta(\emptyset, \emptyset)=0 .
$$

If we consider $p(A, B)=\max \{\delta(A, B) ; \delta(B, A)\}$, with $A, B \subset \mathbb{C}$, void or not, $p$ becomes an "écart" ([4], $\S 1$, Def. 1$)$.

b) The single valued extension property and the decomposability. Let $X$ be a Banach space and let $\mathscr{L}(X)$ be the set of all continuous linear operators on $X$. Consider $T \in \mathscr{L}(X)$.

Let $Y$ be an invariant subspace for $T$. Denote by $\sigma(T)$ the spectrum of $T$, by $T \mid Y$ the restriction of $T$ to the subspace $Y(T \mid Y \in \mathscr{L}(Y))$ and by $\dot{T} \in \mathscr{L}(\dot{X})$ the co-induced operator $T$ on $\dot{X}=X / Y$.

Definition 1.6. (see [5]). The operator $T \in \mathscr{L}(X)$ has the single valued extension property (s. v. e. p.) if for any analytic function $f: D_{f} \rightarrow X\left(D_{f} \subset \mathbb{C}\right.$ open $)$, the identity $(\lambda I-T) f(\lambda) \equiv 0$ on $D_{f}$ implies that $f(\lambda) \equiv 0$ on $D_{f}$.

Denote by $\mathscr{P}(X)$ the set of all operators in $\mathscr{L}(X)$ which have the s.v.e.p.

For $T \in \mathscr{P}(X)$, we say that a point $\lambda_{0} \in \mathbb{C}$ is in the local resolvent $\varrho_{T}(x)$ of $x \in X$ if there is an analytic function $\lambda \rightarrow x_{T}(\lambda)$ (necessarily unique) defined in a neighbourhood of $\lambda_{0}$, with values in $X$, such that $(\lambda I-T) x_{T}(\lambda) \equiv x$.

The local spectrum of $x \in X$ is $\sigma_{T}(x)=\complement \varrho_{T}(x)$.

For $F$ closed in $\mathbb{C}$, we define

$$
X_{T}(F)=\left\{x \in X \mid \sigma_{T}(x) \subset F\right\} .
$$

Definition 1.7. (see [6]). Consider $T \in \mathscr{L}(X)$ and $Y$ an invariant subspace for $T$.

$Y$ is called analytically invariant for $T$ if for every analytic function $f: D_{f} \rightarrow X$ $\left(D_{f} \subset \mathbb{C}\right.$ open), the condition $(\lambda I-T) f(\lambda) \in Y$ on $D_{f}$ implies that $f(\lambda) \in Y$ on $D_{f}$.

$Y$ is called T-absorbing if for any $\lambda \in \sigma(T \mid Y)$ the inclusion $\{x \mid(\lambda I-T) x \in Y\} \subset$ c $Y$ holds. 
$Y$ is called a spectral maximal space of $T$ if for any subspace $Z$ invariant for $T$, the inclusion $\sigma(T \mid Z) \subset \sigma(T \mid Y)$ implies $Z \subset Y$.

Definition 1.8 (see [5]). An operator $T \in \mathscr{L}(X)$ is called decomposable if for every finite open covering $\left\{G_{i}\right\}_{1 \leqq i \leqq n}$ of $\sigma(T)$ there exist $\left\{Y_{i}\right\}_{1 \leqq i \leqq n}$ spectral maximal spaces of $T$ such that $\sigma\left(T \mid Y_{i}\right) \subset G_{i}(1 \leqq i \leqq n)$ and $X=\sum_{i=1}^{n} Y_{i}$.

We will denote by $\mathscr{D}(X)$ the set of decomposable operators.

Note that $\mathscr{D}(X) \subset \mathscr{P}(X)$ (see, for instance, [5], Ch. 2,1.4).

Definition 1.9 (see [8]). For $T_{1}, T_{2} \in \mathscr{L}(X)$, denote

$$
\left(T_{1} \backslash T_{2}\right)^{[n]}=\sum_{k=0}^{n}(-1)^{k} C_{n}^{k} T_{1}^{n-k} T_{2}^{k}, \quad d_{\mathrm{sp}}\left(T_{1}, T_{2}\right)=\varlimsup_{n \rightarrow \infty}\left\|\left(T_{1} \backslash T_{2}\right)^{[n]}\right\|^{1 / n} .
$$

Remark 1.10. In [8], F. H. Vasilescu proved that, if $T_{1}, T_{2} \in \mathscr{D}(X)$, then

$$
d_{\mathrm{sp}}\left(T_{1}, T_{2}\right)=\sup _{x \in X} \sup _{\lambda \in \sigma_{T 1}(x)} \inf _{\mu \in \sigma_{T 2}(x)}|\lambda-\mu| .
$$

In fact, a careful look at the proof of that result shows that the inequality

remains valid for $T_{1}, T_{2} \in \mathscr{P}(X)$.

$$
d_{\mathrm{sp}}\left(T_{1}, T_{2}\right) \geqq \sup _{x \in X} \sup _{\lambda \in \sigma_{T 1}(x)} \inf _{\mu \in \sigma_{T_{2}(x)}}|\lambda-\mu|
$$

Denote $p\left(T_{1}, T_{2}\right)=\max \left\{d_{\mathrm{sp}}\left(T_{1}, T_{2}\right) ; d_{\mathrm{sp}}\left(T_{2}, T_{1}\right)\right\}$ (see [1], [2]).

Definition 1.11. We say that $T_{1}, T_{2} \in \mathscr{L}(X)$ are spectral equivalent (or quasinilpotent equivalent) if $p\left(T_{1}, T_{2}\right)=0$. We denote this by $T_{1} \sim{ }^{\text {sp }} T_{2}$ (see [5], Ch. 1, 2).

Consider $T_{0}, T_{n} \in \mathscr{L}(X)(n \geqq 1)$. We say that the sequence $T_{n}$ converges spectrally to $T_{0}$ if $p\left(T_{n}, T_{0}\right) \rightarrow 0$. We denote this by $T_{n} \rightarrow{ }^{\text {sp }} T_{0}$ (see, for instance, [2]).

c) Analytic residuum. The following notions were introduced in [10].

Definition 1.12. Let $T \in \mathscr{L}(X)$. An open set $\Omega \subset \mathbb{C}$ is a set of analytic uniqueness for $T$ if for any open set $\omega \subset \Omega$ and any analytic function $f_{0}: \omega \rightarrow X$, the identity $(\lambda I-T) f_{0}(\lambda) \equiv 0$ on $\omega$ implies that $f_{0}(\lambda) \equiv 0$ on $\omega$.

Denote by $\Omega_{T}$ the maximal open set of analytic uniqueness for $T$; then $S_{T}=C \Omega_{T}$ is called the analytic residuum of $T$.

Remark 1.13. $S_{T}=\emptyset$ if and only if $T$ has the s.v.e.p. Note also that $S_{T} \subset \sigma(T)$.

Definition 1.14. An analytic function $f_{x}: D_{\boldsymbol{f}} \rightarrow X$ verifying the equation

$$
(\lambda I-T) f_{x}(\lambda)=x\left(\lambda \in D_{f}\right)
$$

is called a $T$-associated function for $x \in X$.

For $x \in X$, denote by $\delta_{T}(x)$ the open set of the points $\lambda_{0} \in \mathbb{C}$ with the property that $\lambda_{0}$ has a neighbourhood where there exists at least one $T$-associated function of $x$. 
Denote

$$
\begin{aligned}
& \gamma_{T}(x)=C \delta_{T}(x), \\
& \varrho_{T}(x)=\delta_{T}(x) \cap \Omega_{T}, \\
& \sigma_{T}(x)=C \varrho_{T}(x)=\gamma_{T}(x) \cup S_{T} .
\end{aligned}
$$

Remark 1.15. The $T$-associated function is not generally unique; however, it is unique on $\varrho_{T}(x)$.

If $T$ has the s.v.e.p., then $\gamma_{T}(x)=\sigma_{T}(x)$ and $\sigma_{T}(x)$ has the usual meaning.

Definition 1.16. For $T \in \mathscr{L}(X)$ and $F$ closed in $\mathbb{C}$, denote

$$
\begin{gathered}
X_{T}(F)=\left\{x \in X \mid \sigma_{T}(x) \subset F\right\}, \\
\tilde{X}_{T}(F)=\left\{x \in X \mid \gamma_{T}(x) \subset F\right\} .
\end{gathered}
$$

\section{WEAK SPECTRAL EQUIVALENCE}

a) The weak spectral pseudo-distance. Definition 2.1. Let $T_{1}, T_{2} \in \mathscr{L}(X)$. We define

$$
\delta_{w}\left(T_{1}, T_{2}\right)=\sup _{x \in X} \delta\left(\sigma_{T_{1}}(x), \sigma_{T_{2}}(x)\right)
$$

and

$$
p_{w}\left(T_{1}, T_{2}\right)=\max \left\{\delta_{w}\left(T_{1}, T_{2}\right) ; \delta_{w}\left(T_{2}, T_{2}\right)\right\} .
$$

Remark 2.2. According to the facts mentioned in Remark 1.10, if $T_{1}$ and $T_{2}$ are decomposable, then $\delta_{w}\left(T_{1}, T_{2}\right)=d_{\text {sp }}\left(T_{1}, T_{2}\right)$ and if they have the s.v.e.p., then $\delta_{w}\left(T_{1}, T_{2}\right) \leqq d_{\mathrm{sp}}\left(T_{1}, T_{2}\right)$. The equality remains true if $T_{2}$ is decomposable and $T_{1}$ is semi-decomposable (i.e. if $T_{1}$ has the s.v.e.p. and for every closed $F, X_{T_{1}}(F)$ is closed) ([12], Cor. 1 of Th. 6.4).

The above mentioned inequality is false if $T_{1}$ has not the s.v.e.p. and $T_{2}$ has this property.

Example. Consider $X=l^{2}, T_{1}$ the adjoint of the unilateral shift, $T_{2}=0$. One shows that $T_{1} \notin \mathscr{P}(x)$ and $S_{T_{1}}=D_{1}$, where $D_{1}$ denotes the unit disc centered at the origin (see Appendix A, 1). Therefore $\sigma_{T_{1}}(x)=D_{1}$ and

It follows that

$$
\sigma_{T_{2}}(x)=\left\{\begin{array}{ccc}
\emptyset & \text { if } & x=0 \\
\{0\} & \text { if } & x \neq 0
\end{array}\right.
$$

hence $\delta_{w}\left(T_{1}, T_{2}\right)=\infty$.

$$
\delta\left(\sigma_{T_{1}}(x), \sigma_{T_{2}}(x)\right)=\left\{\begin{array}{lll}
\delta\left(D_{1}, \emptyset\right)=\infty & \text { if } & x=0, \\
\delta\left(D_{1},\{0\}\right)=1 & \text { if } & x \neq 0,
\end{array}\right.
$$

On the other hand,

$$
d_{\mathrm{sp}}\left(T_{1}, T_{2}\right)=\varlimsup_{n}\left\|\left(T_{1} \backslash T_{2}\right)^{[n]}\right\|^{1 / n}=\left\|T_{1}\right\|_{\mathrm{sp}}=1 .
$$


Remark 2.3. It is easy to see that $p_{w}\left(T_{1}, T_{2}\right)=\infty$ if and only if one of the operators has the s.v.e.p. and the other has not the s.v.e.p. (we use that fact that $\left.\sup _{x \neq 0} \delta\left(\sigma_{T_{1}}(x), \sigma_{T_{2}}(x)\right) \leqq \sup _{\lambda \in \sigma\left(T_{1}\right)} \sup _{\mu \in \sigma\left(T_{2}\right)}|\lambda-\mu|<\infty\right)$.

Remark 2.4. For every $T \in \mathscr{P}(X)$,

(the proof is simple).

$$
\|T\|_{s p}=\delta_{w}(T, 0)
$$

Remark 2.5. In general, $\delta_{w}\left(T_{1}, T_{2}\right) \neq \delta_{w}\left(T_{2}, T_{1}\right)$.

Example. Let $U^{*}$ be the adjoint of the unilateral shift on $l^{2}$ and let $T_{0} \in \mathscr{L}\left(l^{2}\right)$ be such that $\sigma\left(T_{0}\right)=\left\{\lambda_{0}\right\}$ with $\lambda_{0} \notin D_{1}=\{\lambda \in \mathbb{C}|| \lambda \mid \leqq 1\}$.

We know that $\sigma_{U^{*}}(x)=D_{1}$ for all $x \in l^{2}$. We obviously have $\sigma_{T_{0}}(x)\left\{\lambda_{0}\right\}$ for all $x \in l^{2} \backslash\{0\}$ and $\sigma_{T_{0}}(0)=\emptyset$. Therefore

and

$$
\begin{gathered}
\delta_{w}\left(U^{*}, T_{0}\right)=\sup _{x \in l^{2}} \sup _{\lambda \in \sigma_{U^{*}(x)}} \inf _{\mu \in \sigma_{T_{0}}(x)}|\lambda-\mu|= \\
=\max \left\{\sup _{\substack{x \in l^{2} \\
x \neq 0}} \sup _{\lambda \in D_{1}}\left|\lambda-\lambda_{0}\right| ; \delta\left(D_{1}, \emptyset\right)\right\}=\max \left\{\sup _{\lambda \in D_{1}}\left|\lambda-\lambda_{0}\right| ; \infty\right\}=\infty
\end{gathered}
$$

$$
\begin{gathered}
\delta_{w}\left(T_{0}, U^{*}\right)=\sup _{x \in l^{2}} \sup _{\mu \in \sigma_{T 0}(x)} \inf _{\lambda \in \sigma \sigma^{*}(x)}|\lambda-\mu|= \\
=\max \left\{\sup _{\substack{x \in l^{2} \\
x \neq 0}} \inf _{\lambda \in D_{1}}\left|\lambda-\lambda_{0}\right| ; \delta\left(\emptyset, D_{1}\right)\right\}=\max \left\{\inf _{\lambda \in D_{1}}\left|\lambda-\lambda_{0}\right| ; 0\right\}=\inf _{\lambda \in D_{1}}\left|\lambda-\lambda_{0}\right| .
\end{gathered}
$$

Therefore, we obtain $\delta_{w}\left(T_{0}, U^{*}\right)<\delta_{w}\left(U^{*}, T_{0}\right)$.

Lemma 2.6. For $T_{1}, T_{2}, T_{3} \in \mathscr{L}(X)$, the triangle inequality holds:

$$
p_{w}\left(T_{1}, T_{3}\right) \leqq p_{w}\left(T_{1}, T_{2}\right)+p_{w}\left(T_{2}, T_{3}\right) .
$$

Proof. It results immediately from Lemma 1.3 and the subadditivity of the supremum.

Remark 2.7. $p_{w}$ is a semi-metric (it is not a metric: $p_{w}(T, 0)=\|T\|_{\mathrm{sp}}=0$ for $T$ quasi-nilpotent, $T \neq 0$ ).

b) The weak spectral equivalence. Definition 2.8. Let $T_{1}, T_{2} \in \mathscr{L}(X)$. We say that $T_{1}$ is weakly spectrally equivalent to $T_{2}$ if

$$
p_{w}\left(T_{1}, T_{2}\right)=0 .
$$

We denote this by $T_{1} \sim^{w} T_{2}$.

It is clear that $\sim^{w}$ is an equivalence relation.

Remark 2.9. If $T_{1}$ and $T_{2}$ have the s.v.e.p., then the spectral equivalence $T_{1} \sim^{s p} T_{2}$ 
implies the weak spectral equivalence $T_{1} \sim{ }^{w} T_{2}$; we shall prove later (Theorem 2.21) that this fact is true for any operators in $\mathscr{L}(X)$. If $T_{1}$ and $T_{2}$ are decomposable, the two notions are equivalent (cf. Remark 2.2).

Remark 2.10. $T_{1} \sim^{w} T_{2}$ means that, for every $x \in X, p\left(\sigma_{T_{1}}(x), \sigma_{T_{2}}(x)\right)=0 ; \sigma_{T_{1}}(x)$ being compact, it follows from Lemma 1.2, b) that $T_{1} \sim{ }^{w} T_{2}$ is equivalent to $\sigma_{T_{1}}(x)=$ $=\sigma_{T_{2}}(x)$ for every $x \in X$.

Proposition 2.11. a) If $S_{T_{1}}=S_{T_{2}}$ and $\gamma_{T_{1}}(x)=\gamma_{T_{2}}(x)$ for every $x \in X$, then $T_{1} \sim{ }^{w} T_{2}$.

b) Conversely, if $T_{1} \sim{ }^{w} T_{2}$, then $S_{T_{1}}=S_{T_{2}}$.

If, moreover, $\gamma_{T_{1}}(x) \cap S_{T_{1}}=\gamma_{T_{2}}(x) \cap S_{T_{2}}$ for every $x \in X$, then $\gamma_{T_{1}}(x)=\gamma_{T_{2}}(x)$ for every $x \in X$.

Proof. a) is obvious, by the definition of $\sigma_{T_{2}}(x)$ and Remark 2.10 .

b) We know that $\gamma_{T_{1}}(x) \cup S_{T_{1}}=\gamma_{T_{2}}(x) \cup S_{T_{2}}$ for every $x \in X$, in particular for $x=0$. But $\gamma_{T_{i}}(0)=\emptyset$ (obviously $\delta_{T}(0)=\mathbb{C}$ for $T \in \mathscr{L}(x)$ ). Hence $S_{T_{1}}=S_{T_{2}}$.

It is known that $A \cup B=A \cup C$ and $A \cap B=A \cap C$ imply that $B=C$. We take $A=S_{T_{1}}=S_{T_{2}}, B=\gamma_{T_{1}}(x), C=\gamma_{T_{2}}(x)$ and the statement follows.

This proposition has the following immediate interestıng

Corollary 2.12. Let $T_{1}, T_{2} \in \mathscr{L}(X)$. If $T_{1} \sim^{w} T_{2}$ and $T_{1}$ has the s.v.e.p., then $T_{0}$ has the s.v.e.p. as well.

Proposition 2.13. Let $T_{1}, T_{2} \in \mathscr{L}(X)$. If $T_{1} \sim^{w} T_{2}$, then

$$
\sigma\left(T_{1}\right)=\sigma\left(T_{2}\right) .
$$

Proof. For every $x \in X, \sigma_{T_{1}}(x)=\sigma_{T_{2}}(x)$. Hence by [10], Prop. 2.4, Cor. 1,

$$
\sigma\left(T_{1}\right)=\bigcup_{x \in X} \sigma_{T_{1}}(x)=\bigcup_{x \in X} \sigma_{T_{2}}(x)=\sigma\left(T_{2}\right) .
$$

Proposition 2.14. Let $T_{1}, T_{2} \in \mathscr{L}(X)$ and let $F$ be closed in $\mathbb{C}$.

a) If $T_{1} \sim{ }^{w} T_{2}$, then

$1^{\circ} X_{T_{1}}(F)=X_{T_{2}}(F)$;

$2^{\circ} \tilde{X}_{T_{1}}(F)=\tilde{X}_{T_{2}}(F)$ in each of the following cases:

a) $S_{T_{i}} \subset F \quad(i=1,2)$,

及) $\gamma_{T_{1}}(x) \cap S_{T_{1}}=\gamma_{T_{2}}(x) \cap S_{T_{2}} \quad(\forall) x \in X$

b) Conversely, if $X_{T_{1}}(F)=X_{T_{2}}(F)$ for every $F$ closed in $\mathbb{C}$, then $T_{1} \sim^{w} T_{2}$.

Proof. a) $1^{\circ}$ Obvious.

$\left.2^{\circ} \propto\right)$ Since $S_{T_{i}} \subset F, \tilde{X}_{T_{i}}(F)=X_{T_{i}}(F)$ and one applies $1^{\circ}$.

及) By Proposition $2.11, b)$

b) Obviously, $x \in X_{T_{2}}\left(\sigma_{T_{2}}(x)\right)$ for every $x \in X$. Therefore, by hypothesis, $x \in$ 
$\in X_{T_{1}}\left(\sigma_{T_{2}}(x)\right)$, that is, from the definition of $X_{T_{1}}(),. \sigma_{T_{1}}(x) \subset \sigma_{T_{2}}(x)$. The other inclusion can be proved in the same way.

Remark 2.15. A result similar to point b) was obtained for the spectral equivalence ([5], Ch. 2, Th. 2.2), but only in the case of decomposable operators (in this case, the two notions of the equivalence coincide).

Theorem 2.16. Let $T_{1}, T_{2} \in \mathscr{L}(X)$. If $T_{1} \sim^{w} T_{2}$ abd $T_{1}$ is decomposable, then $T_{2}$ is decomposable as well.

Proof. The proof will proceed in several steps.

a) By Corollary 2.12, $T_{1}$ and $T_{2}$ have the s.v.e.p..

b) $1^{\circ}$ By Proposition 2.14, a),$X_{T_{1}}(F)=X_{T_{2}}(F)$ for every $F$ closed in $\mathbb{C}$. Denote $Y_{F}=X_{T_{1}}(F)=X_{T_{2}}(F)$.

$2^{\circ} Y_{F}=X_{T_{2}}(F)$ is invariant for $T_{2}$; hence $T_{2}\left(Y_{F}\right) \subset Y_{F}$.

$3^{\circ}$ Because $T_{1}$ is decomposable, $Y_{F}=X_{T_{1}}(F)$ is a spectral maximal space for $T_{1}$ for every $F$ closed, $F \subset \sigma\left(T_{1}\right)([5]$, Ch. 2, Th. 1.5) hence for every $F$ closed in $\mathbb{C}$. Indeed $X_{T_{1}}(F)=X_{T_{1}}\left(F \cap \sigma\left(T_{1}\right)\right)\left([5]\right.$, Ch. 1, 1.1) and $F \cap \sigma\left(T_{1}\right) \subset \sigma\left(T_{1}\right)$.

Therefore $Y_{F}$ is closed. On the other hand, $Y_{F}=X_{T_{2}}(F)$ and $T_{2}$ has the s.v.e.p.. It follows $\left([5]\right.$, Ch. 1, Prop. 3.8) that $Y_{F}$ is a spectral maximal space for $T_{2}$ and that $\sigma\left(T_{2} \mid Y_{F}\right) \subset F$.

$4^{\circ}$ It follows that

$$
\begin{array}{lll}
\sigma\left(T_{2} \mid Y_{F}\right) \subset F & (\forall) F & \text { closed (from } \left.3^{\circ}\right) \\
T_{2}\left(Y_{F}\right) \subset Y_{F} & (\forall F) & \text { closed (from } \left.2^{\circ}\right) .
\end{array}
$$

Hence ([5], Ch. 2, Th. 2.6) $T_{2}$ is decomposable.

Proposition 2.17. Let $T_{1}, T_{2}^{\prime} \in \mathscr{L}(X)$ and let $f: D \rightarrow \mathbb{C}$ be an analytic function defined on a neighbourhood of $\sigma\left(T_{1}\right) \cup \sigma\left(T_{2}\right)$, nonconstant on every component of $D$.

If $T_{1} \sim{ }^{w} T_{2}$, then $f\left(T_{1}\right) \sim{ }^{w} f\left(T_{2}\right)$.

Proof. From [11], III, 3.15 and 3.17, it follows that $f\left(\gamma_{T_{i}}(x)\right)=\gamma_{f\left(T_{i}\right)}(x)(x \in X)$ and $f\left(S_{T_{i}}\right)=S_{f\left(T_{i}\right)}(i=1,2)$. Therefore

$$
\begin{gathered}
\sigma_{f\left(T_{i}\right)}(x)=\gamma_{f\left(T_{i}\right)}(x) \cup S_{f\left(T_{i}\right)}=f\left(\gamma_{T_{i}}(x)\right) \cup f\left(S_{T_{i}}\right)= \\
=f\left(\gamma_{T_{i}}(x) \cup S_{T_{i}}\right)=f\left(\sigma_{T_{i}}(x)\right) \quad(i=1,2)
\end{gathered}
$$

and $\sigma_{T_{1}}(x)=\sigma_{T_{2}}(x)$ implies that $\sigma_{f\left(T_{1}\right)}(x)=\sigma_{f\left(T_{2}\right)}(x)$ for every $x \in X$.

Now we show how the similarity preserves the local spectral properties.

Lemma 2.18. Let $T \in \mathscr{L}(X)$ and let $U$ be an invertible operator in $\mathscr{L}(X)$. The following equalities hold:

a) $S_{T}=S_{U^{-1} T U}$,

b) $\gamma_{T}(U x)=\gamma_{U^{-1} T U}(x)(\forall) x \in X$. 
Proof. a) Let $\omega \subset \Omega_{T}$ and let $f: \omega \rightarrow X$ bz an analytic function with $\left(\lambda I-U^{-1} T U\right) f(\lambda) \equiv 0$ on $\omega$. Hence $U^{-1}(\lambda I-T) \cup f(\lambda) \equiv 0$ on $\omega$ and, $U^{-1}$ being injective, $(\lambda I-T) \cup f(\lambda) \equiv 0$ on $\omega$.

Define $f_{U}: \omega \rightarrow X$ by $f_{U}(\lambda)=U f(\lambda)$. It is easy to prove that $f_{U}$ is an analytic function. Moreover, $(\lambda I-T) f_{U}(\lambda) \equiv 0$ on $\omega ; \omega$ is included in $\Omega_{T}$, so that $f_{U}(\lambda) \equiv 0$ on $\omega$. By the injectivity of $U$ we obtain $f(\lambda) \equiv 0$ on $\omega$. Hence $\left(v \subset \Omega_{U^{-1} T U}\right.$, so that $\Omega_{T} \subset \Omega_{U^{-1} T U}$.

The converse inclusion can be proved in the same way, using $f_{U}(\lambda)=U^{-1} f(\lambda)$.

b) We prove that $\delta_{T}(U x)=\delta_{U^{-1} T U}(x)$.

Let $\lambda_{0} \in \delta_{T}\left(U_{\lambda}\right)$ and let $D$ be a neighbourhood of $\lambda_{0}$, for which there exists an analytic function $f: D \rightarrow X$ with $(\lambda I-T) f(\lambda) \equiv U x$ on $D$, or $U^{-1}(\lambda I-T) f(\lambda) \equiv$ $\equiv x$ on $D$. Define $f_{U}: D \rightarrow X$ by $f_{U}(\lambda)=U^{-1} f(\lambda) ; f_{U}$ is an analytic function and $U^{-1}(\lambda I-T) \cup f_{U}(\lambda) \equiv x$ on $D$, or $\left(\lambda I-U^{-1} T U\right) f_{U}(\lambda) \equiv x$ on $D$. Therefore $\lambda_{0} \in \delta_{U^{-1} T U}(x)$.

In the same way the converse inclusion can be proved.

Corollary 2.19. Let $T, U \in \mathscr{L}(X), U$ invertible. We have

$$
\sigma_{T}(U x)=\sigma_{U^{-1} T U}(x) \quad(\forall) x \in X .
$$

Proposition 2.20. Let $T_{1}, T_{2} \in \mathscr{L}(X)$ and let $U \in \mathscr{L}(X)$ be invertible. If $T_{1} \sim^{w} T_{2}$ then $U^{-1} T_{1} U \sim{ }^{w} U^{-1} T_{2} U$.

Proof. By hypothesis, $\sigma_{T_{1}}(x)=\sigma_{T_{2}}(x)$ for every $x \in X$, therefore $\sigma_{T_{1}}(U x)=$ $=\sigma_{T_{2}}(U x)$ for every $x \in X$. From Corollary 2.19 we obtain $\sigma_{U^{-1} T_{1} U}(x)=\sigma_{U^{-1} T_{2} U}(x)$ for every $x \in X$, that is, $U^{-1} T_{1} U \sim^{w} U^{-1} T_{2} U$.

We observe that in [5], Ch. $1, \S 2$ we can separate the existence from the uniqueness of the extension of the resolvent; we obtain

Theorem 2.21. If $T_{1}, T_{2} \in \mathscr{L}(X)$ and $T_{1} \sim{ }^{\text {sp }} T_{2}$, then $T_{1} \sim^{w} T_{2}$.

Proof. We shall prove that $\gamma_{T_{1}}(x)=\gamma_{T_{2}}(x)$ for every $x \in X$ and that $S_{T_{1}}=S_{T_{2}}$.

a) $\gamma_{T_{1}}(x)=\gamma_{T_{2}}(x)$ forllos as in [5], Ch. 1, Th. 2.4.

Let $\lambda_{0} \in \delta_{T_{1}}(x)$; there exist an open neighbourhood $D$ of $\lambda_{0}$ and an analytic function $x_{1}: D \rightarrow X$ such that $\left(\lambda I-T_{1}\right) x_{1}(\lambda)=x$ on $D$. We take $D_{0} \subset \delta_{T_{1}}(x)$ and construct an analytic function $x_{2}$ on $D$ with $\left(\partial I-T_{2}\right) x_{2}(\lambda)=x$ on $D$. It follows that $\lambda_{0} \in$ $\in \delta_{T_{2}}(x)$.

The other inclusion is proved in the same way.

b) In order to prove that $S_{T_{1}}=S_{T_{2}}$, we use the proof of The. 2.3, Ch. 1 from [5].

Let $D_{f} \subset \Omega_{T_{1}}$ and let $f: D_{f} \rightarrow X$ be an analytic function with $\left(\lambda I-T_{2}\right) f(\lambda) \equiv 0$ on $D_{f}$. One constructs, for every $\lambda_{0} \in D_{f}$, an analytic function $g_{\lambda}$ on $\mathbb{C} \backslash\left\{\lambda_{0}\right\}$ which verifies $\left(\mu I-T_{1}\right) g_{\lambda}(\mu)=f\left(\lambda_{0}\right)$ and puts

$$
h_{\lambda}(\mu)=\frac{1}{2 \pi \mathrm{i}} \int_{\left|\xi-\lambda_{0}\right|=r_{0}} \frac{g_{\xi}(\mu)}{\xi-\lambda} \mathrm{d} \xi^{*}
$$


which is an analytic function on Int $D\left(\lambda_{0}, r_{0}\right) \subset D_{f}$; in particular, $h_{\lambda_{0}}$ is an analytic function and verifies $\left(\mu I-T_{1}\right) h_{\lambda_{0}}(\mu)=f\left(\lambda_{0}\right)$ on Int $D\left(\lambda_{0}, r_{0}\right)$.

Now, $D_{f} \subset \Omega_{T_{1}}$ and $\left(\right.$ Int $\left.D\left(\lambda_{0}, r_{0}\right)\right) \backslash\left\{\lambda_{0}\right\} \subset D_{f}$. It follows that $g_{\lambda_{0}}=h_{\lambda_{0}}$ on the open connected set $\left(\right.$ Int $\left.D\left(\lambda_{0}, r_{0}\right)\right) \backslash\left\{\lambda_{0}\right\}$, therefore we can construct an analytic function

$$
f_{\lambda_{0}}^{0}(\mu)=\left\{\begin{array}{l}
g_{\lambda_{0}}(\mu) \text { for } \mu \neq \lambda_{0}, \\
h_{\lambda_{0}}(\mu) \text { for } \mu=\lambda_{0},
\end{array}\right.
$$

which verifies $\left(\mu I-T_{1}\right) f_{\lambda_{0}}^{0}(\mu) \equiv f\left(\lambda_{0}\right)$ on all $\mathbb{C}$. By [7], 2.1(c), $f_{\left(\lambda_{0}\right)}$ must be 0 .

$\lambda_{0}$ being arbitrary, $f(\lambda) \equiv 0$ on $D_{f}$, hence $D_{f} \subset \Omega_{T_{2}}$.

In conclusion, $\Omega_{T_{1}} \subset \Omega_{T_{i}}$, the other inclusion being proved in the same manner.

c) Properties of permanence of the local spectrum. In order to obtain some results concerning the permanence of the weak spectral equivalence by passing to subspaces and quotient spaces, we are interested in the behaviour of the local spectrum in these cases.

It is known that, if $T \in \mathscr{L}(X)$ and $Y$ is an invariant subspace for $T$, then

$1^{\circ} S_{T \mid Y} \subset S_{T} ; \gamma_{T \mid Y}(x) \supset \gamma_{T}(x)(x \in X)$;

$2^{\circ} S_{T} \subset S_{T} \cup \sigma(T \mid Y) ; S_{T} \subset S_{T} \cup \sigma(T \mid Y) ; \gamma_{T}(\dot{x}) \subset \gamma_{T}(x) \subset \gamma_{T}(\dot{x}) \cup \sigma(T \mid Y)$ $(x \in X)$ (see for instance [3].1.1).

First we shall study conditions upon which the equality $\sigma_{T \mid Y}(x)=\sigma_{Y}(x)$ holds for $x \in Y$.

For this purpose we introduce a notion which generalizes the notion of the analytically invariant subspace.

Definition 2.22. Let $T \in \mathscr{L}(X)$ and let $Y$ be an invariant subspace for $T$. Let $\Omega$ be an open set in $\mathbb{C}$. We say that $Y$ is $\Omega$-analytically invariant for $T$ if for every open set $\omega \subset \Omega$ and every analytic function $f: \omega \rightarrow X$ which verifies $(\lambda I-T) f(\lambda) \in Y$ for every $\lambda \in \omega$, the function $f$ is $Y$-valued.

Remark 2.23. A $\mathbb{C}$-analytically invariant subspace is analytically invariant for $T$.

Remark 2.24. The subspace $\{0\}$ is $\Omega$-analytically invariant for $T$ if and only if $\Omega$ is a set of analytic uniqueness for $T$ (see Def. 1.12). The subspace $Y$ is $\Omega$-analytically invariant for $T$ if and only if $\Omega$ is a set of analytic uniqueness for $\dot{T} \in \mathscr{L}(X / Y)$.

Examples. 1) If $Y$ is an invariant subspace for $T$, then $Y$ is $\varrho(\dot{T})$-analytically invariant for $T$.

Indeed, let $\omega \subset \varrho(\dot{T})$ and let $f: \omega \rightarrow X$ be an analytic function with $(\lambda I-T) f(\lambda) \in Y$ for every $\lambda \in \omega$. Now $\lambda \in \omega \subset \varrho(\dot{T})$ and $(\lambda \dot{I}-\dot{T}) \dot{f(\lambda)}=\dot{O}$ imply $\dot{f(\lambda)}=\dot{O}$, that is, $f(\lambda) \in Y$.

2) Denote by A the example from [6], 2.28, that is:

Let $U^{*}$ be the adjoint of the unilateral shift on $l^{2}$ and let $\lambda \in \mathbb{C} \backslash\{0\}$ with $|\lambda|<\frac{1}{2}$. 
Denote by $E_{\lambda}$ the eigenspace corresponding to the eigenvalue $\lambda$. We know (see Appendix A, 1) that $\Omega_{\mathbf{L}^{*}}=\{\lambda|| \lambda \mid>1\}$.

$E_{\lambda}$ is not analytically invariant, but it is $\Omega_{U^{*}}$-analytically invariant.

The first statement is proved in [6]. Let us prove the second one.

Let $\omega \subset \Omega_{U^{*}}$ and let $f: \omega \rightarrow l^{2}$ be analytic and such that $\left(\mu I-U^{*}\right) f(\mu) \in E_{\lambda}$ on $\omega$. We fix $\mu \in \omega$ and denote $f(\mu)=\left\{x_{n}\right\}_{n \geqq 0}$. Hence $\mu x_{n-1}-x_{n}=-r \lambda^{n-1}(n \geqq 1$, $r \in \mathbb{C}$ ), so that

$$
x_{n}=\frac{1}{\mu-\lambda}\left[\mu^{n+1} x_{0}+\left(r-\lambda x_{0}\right) \mu^{n}-r \lambda^{n}\right] .
$$

Because $\left\{x_{n}\right\} \in l^{2}$, it follows that $\left|x_{n}\right|^{2} \rightarrow 0 ; \mu$ being of modulus greater than 1 , we have $x_{0}=0$ and $r=0$. Hence $f(\mu)=0 \in E_{\lambda}$.

The following result is a generalization of ([6], Theorems 3.7 and 2.26) which prove that a spectral maximal space is analytically invariant for $T$, if $T$ has the s.v.e.p..

Proposition 2.25. If $T \in \mathscr{L}(X)$ and $Y$ is a spectral maximal space for $T$, then $Y$ is $\Omega_{T^{-}}$analytically invariant for $T$.

Proof. $Y$ is $T$-absorbing, cf. [6], Th. 3.7. Now the proof follows that of Th. 2.26 from [6].

Let $\omega \subset \Omega_{T}$ and let $f: \omega \rightarrow X$ be an analytic function with $(\lambda I-T) f(\lambda) \in Y$ on $\omega$.

Because $Y$ is $T$-absorbing, it follows that $f(\lambda) \in Y$ for $\lambda \in \sigma(T \mid Y)$.

Now let $\lambda \in \varrho(T \mid Y) \cap \omega$. There exists $(\lambda I-T \mid Y)^{-1}$. Denote

$$
y(\lambda)=(\lambda I-T) f(\lambda) \in Y
$$

$(\lambda I-T)^{-1} y(\lambda)$ makes sense and is in $Y$.

We have

$$
y(\lambda)=(\lambda I-T)(\lambda I-T)^{-1} y(\lambda), \quad y(\lambda)=(\lambda I-T) f(\lambda) .
$$

It follows that $(\lambda I-T)\left[f(\lambda)-(\lambda I-T)^{-1} y(\lambda)\right]=0$ for $\lambda \in \omega \cap \varrho(T \mid Y) \subset$ $\subset \Omega_{T}$. Hence $f(\lambda)=(\lambda I-T)^{-1} y(\lambda) \in Y$,

Proposition 2.26. Let $T \in \mathscr{L}(X)$ and let $Y$ be an invariant subspace for $T$. If $Y$ is $\Omega_{T}$-analytically invariant for $T$, then for every $x \in Y$,

$$
\sigma_{T \mid Y}(x) \subset \sigma_{T}(x) .
$$

Proof. We prove that $\varrho_{T}(x) \subset \varrho_{T \mid Y}(x)$, that is $\Omega_{T} \cap \delta_{T}(x) \subset \Omega_{T \mid Y} \cap \delta_{T \mid Y}(x)$. We know that $\Omega_{T} \subset \Omega_{T \mid Y}$.

Let $G$ be a connected component of $\delta_{T}(x)$; we know that $G \subset \Omega_{T}$ or $G \subset S_{T}$. We take $G \subset \delta_{T}(x) \cap \Omega_{T} \subset \Omega_{T \mid Y}$; let us show that $G \subset \delta_{T \mid Y}(x)$. Because $G \subset \Omega_{T}$, there exists a unique analytic function $f: G \rightarrow X$ satisfying $(\lambda I-T) f(\lambda)=x \in Y$. Now $G \subset \Omega_{T}$ and $Y$ is $\Omega_{T}$-analytically invariant, therefore $f(\lambda) \in Y$. In other words, $f: G \rightarrow Y$ verifies the required relation, hence $G \subset \delta_{T \mid Y}(x)$.

Proposition 2.27. Let $T \in \mathscr{L}(x)$ and let $Y$ be an invariant subspace for $T$. If $Y$ is 
$\Omega_{T^{-}}$analytically invariant for $T$ and $\Omega_{T \mid Y}=\Omega_{T}$, then for every $x \in Y$,

$$
\sigma_{T \mid Y}(x)=\sigma_{T}(x) \text {. }
$$

Proof. By the previous proposition, $\left.\varrho_{T}(x) \subset \varrho_{T}\right|_{Y}(x)$. Now, $\varrho_{T \mid Y}(x)=\delta_{T \mid Y}(x) \cap$ $\cap \Omega_{T \mid Y}=\delta_{T \mid Y}(x) \cap \Omega_{T} \subset \delta_{T}(x) \cap \Omega_{T}=\varrho_{T}(x)$, using the hypothesis and the inclusion $\delta_{T \mid Y}(x) \subset \delta_{T}(x)$.

We now give an example showing that without the additional hypothesis in Proposition 2.27 (i.e. $\Omega_{T \mid Y}=\Omega_{T}$ ) the equality $\sigma_{T \mid Y}(x)=\sigma_{T}(x)$ may fail.

We use Example A.

It is easy to prove that $S_{U^{*} \mid E_{\lambda}}=\emptyset, \sigma\left(U^{*} \mid E_{\lambda}\right)=\{\lambda\}$ and therefore $\left.\sigma_{U^{*}}\right|_{E_{\lambda}}(x) \cong\{\lambda\}$. On the other hand, $\sigma_{U^{*}}(x)=D_{1}$ for every $x \in E_{\lambda}$. Hence $\sigma_{U^{*} \mid E_{\lambda}}(x) \stackrel{\subsetneq}{\ddagger} \sigma_{U^{*}}(x)$. Note

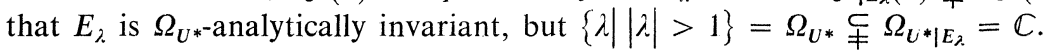

Corollary 2.28. If $T$ has the s.v.e.p. and $Y$ is analytically invariant for $T$, then

for every $x \in X$.

$$
\sigma_{T \mid Y}(x)=\sigma_{T}(x)
$$

Proof. $\Omega_{T}=\mathbb{C}=\Omega_{T \mid Y}$ and $Y$ is $\mathbb{C}$-analytically invariant.

Example. Denote by $\mathrm{B}$ the following example. Let $K$ be a compact set in $\mathbb{C}$, $\mathscr{B}(K)=\{f: K \rightarrow \mathbb{C} \mid f$ bounded $\}, \mathscr{C}(K)=\{f: K \rightarrow \mathbb{C} \mid f$ continuous $\}$. Let $M_{x}$ be the multiplication by $x$ in $\mathscr{B}(K): M_{x} f(x)=x f(x)$ for every $x \in K$.

We can show that $M_{x} \in \mathscr{P}(\mathscr{B}(K)), \mathscr{C}(K)$ is analytically invariant for $M_{x}$ and for every $f \in \mathscr{C}(K)$,

(see Appendix B, 1-4).

$$
\sigma_{\boldsymbol{M}_{\boldsymbol{x}} \mid \mathscr{G}(K)}(f)=\sigma_{\boldsymbol{M}_{\boldsymbol{x}}}(f)=\operatorname{supp} f
$$

Proposition 2.29. Let $T \in \mathscr{L}(X)$, let $Y$ be an invariant subspace for $T$ and $x \in Y$. If

$$
\begin{array}{ll}
1^{\circ} & S_{T \mid Y}=S_{T} \cap \sigma(T \mid Y), \\
2^{\circ} & \gamma_{T \mid Y}(x)=\gamma_{T}(x), \\
3^{\circ} & \sigma_{T}(x) \subset \sigma(T \mid Y) \cup \gamma_{T}(x)
\end{array}
$$

then

$$
\sigma_{T \mid Y}(x)=\sigma_{T}(x) .
$$

Proof. Recall that we always have $\delta_{T \mid Y}(x) \subset \delta_{T}(x)$ and $\Omega_{T \mid Y} \supset \Omega_{T}$. We have to prove that $\varrho_{T \mid Y}(x)=\varrho_{T}(x)$, that is, $\delta_{T \mid Y}(x) \cap \Omega_{T \mid Y}=\delta_{T}(x) \cap \Omega_{T}$.

Let us prove that $\delta_{T \mid Y}(x) \cap \Omega_{T \mid Y} \subset \delta_{T}(x) \cap \Omega_{T}$.

Let $\lambda \in \delta_{T \mid Y}(x) \cap \Omega_{T \mid Y}$; we have $\lambda \in \delta_{T}(x) \cap \Omega_{T \mid Y}$, and we have to show that $\lambda \in \Omega_{T}$.

If $\lambda \in \varrho(T \mid Y)$, then by $3^{\circ}\left(\varrho_{T}(x) \supset \varrho(T \mid Y) \cap \delta_{T}(x)\right)$ we obtain $\lambda \in \varrho_{T}(x)=\delta_{T}(x) \cap \Omega_{T}$, hence $\lambda \in \Omega_{T}$.

If $\lambda \in \sigma(T \mid Y)$ and we suppose that $\lambda \notin \Omega_{T}$, that is $\lambda \in S_{T}$, by $1^{\circ}, \lambda \in S_{T \mid Y}$, which is false. 
In order to prove the reverse inclusion, let $\lambda \in \delta_{T}(x) \cap \Omega_{T}$; we have $\lambda \in \delta_{T}(x) \cap$ $\cap \Omega_{T \mid Y}$; by $2^{\circ}, \lambda \in \delta_{T \mid Y}(x) \cap \Omega_{T \mid Y}$.

The example described right after Proposition 2.27 shows that condition $3^{\circ}$ cannot be omitted in Proposition 2.29.

Remark 2.30. Note that Proposition 2.29 gives another proof of Corollary 2.28.

Remark 2.31. Condition $2^{\circ}$ in Proposition 2.29, $\gamma_{T \mid Y}(x)=\gamma_{T}(x)(x \in Y)$, is fulfilled if the set of values of every function $T$-associated with $x$ is contained in $Y$.

This inclusion holds if $Y$ is a spectral maximal space for $T$ (the proof is similar to that of [5], Ch. 1, Prop. 3.5).

Since in Example $\mathrm{A}$ condition $2^{\circ}$ is not fulfilled, we conclude that $E_{\lambda}$ is not a spectral maximal space for $U^{*}$. In fact, we can prove this directly. Let $\lambda \in \mathbb{C} \backslash\{0\}$ with $|\lambda|<\frac{1}{2}, t_{0}=\lambda, t_{n+1}=\lambda t_{n}+\lambda^{n}$. Hence $\left\{t_{n}\right\}_{n \geqq 0} \in l^{2} \backslash E_{\lambda}$ (see [6], Ex. 2.28). Consider the subspace of $l^{2}$ defined by

$$
Z=\left\{r\left\{\lambda^{n}\right\}_{n \geqq 0}+\alpha\left\{t_{n}\right\}_{n \geqq 0} \mid r, \alpha \in \mathbb{C}\right\} .
$$

We show that $Z$ is invariant for $U^{*}, \sigma\left(U^{*} \mid Z\right)=\{\lambda\}=\sigma\left(U^{*} \mid E_{\lambda}\right)$, but $Z \underset{\nexists}{\supsetneq} E_{\lambda}$ (see Appendix, A, 2).

We now study condition under which the equality $\sigma_{T}(\dot{x})=\sigma_{T}(x)$ holds for a fixed $x \in X$.

Proposition 2.32. Let $T \in \mathscr{L}(X)$, let $Y$ be an invariant subspace for $T$ and $x \in X$.

$1^{\circ}$ If $\sigma(T \mid Y) \subset \sigma_{T}(x)$, then $\sigma_{T}(\dot{x}) \subset \sigma_{T}(x)$.

$2^{\circ}$ If $\sigma(T \mid Y) \subset \sigma_{T}(\dot{x})$, then $\sigma_{T}(x) \subset \sigma_{T}(\dot{x})$.

$3^{\circ}$ If $\sigma(T \mid Y) \subset \sigma_{T}(x) \cap \sigma_{T}(\dot{x})$, then $\sigma_{T}(\dot{x})=\sigma_{T}(x)$.

Proof. $1^{\circ}$ We prove that $\varrho_{T}(x) \subset \varrho_{T}(\dot{x})$.

Let $\lambda_{0} \in \varrho_{T}(x)$, that is, $\lambda_{0} \notin \sigma_{T}(x)$; by hypothesis, $\lambda_{0} \in \varrho(T \mid Y)$, hence there exists $(\lambda I-T)^{-1}$ for $\lambda$ in a neighbourhood $D_{0}$ of $\lambda_{0}$. We want to show that $\lambda_{0} \in \varrho_{T}(\dot{x})=$ $=\delta_{T}(\dot{x}) \cap \Omega_{T}$.

Let $D \subset D_{0}$ be an open neighbourhood of $\lambda_{0}$ and $\dot{g}: D \rightarrow \dot{X}$ an analytic function with $(\lambda \dot{I}-\dot{T}) \dot{g}(\lambda)=\dot{O}$. Therefore $(\lambda I-T) g(\lambda) \in Y$ and $g(\lambda)=(\lambda I-T \mid Y)^{-1}$. . $(\lambda I-T) g(\lambda) \in Y$ by hypothesis. It follows that $g(\lambda) \in Y$ or $g^{\prime}(\lambda)=\dot{O}$. Hence $\lambda_{0} \in \Omega_{T}$.

As $\lambda_{0} \in \delta_{T}(x)$, it follows that there exists an analytic function $f: V_{\lambda_{0}} \rightarrow X$ with $(\lambda I-T) f(\lambda)=x$ on $V_{\lambda_{0}}$. Therefore $\dot{f}: V_{\lambda_{0}} \rightarrow \dot{X}$, defined by $\dot{f}(\lambda)=\dot{f(\lambda)}$, is an analytic function and it verifies $(\lambda \dot{I}-\dot{T}) \dot{f}(\lambda)=\dot{x}$ on $V_{\lambda \cdot 0}$.

(If $x \in Y$, then $\delta_{\dot{T}}(\dot{x})=\mathbb{C}$, so that the second part of the proof is not necessary.)

$2^{\circ}$ Let $\lambda_{0} \notin \sigma_{T}(\dot{x})$; we know that there exists $(\lambda I-T \mid Y)^{-1}$ on a neighbourhood $D_{0}$ of $\lambda_{0}$. We have to show that $\lambda_{0} \in \varrho_{T}(x)=\delta_{T}(x) \cap \Omega_{T}$.

Let $g: D \rightarrow X$ be an analytic function on a neighbourhood $D \subset D_{0}$ of $\lambda_{0}$ with 
$(\lambda I-T) g(\lambda)=0$. By hypothesis, we can write $g(\lambda)=(\lambda I-T)^{-1}(\lambda I-T) g(\lambda)=$ $=0$, so that $g(\lambda)=0$. It follows that $\lambda_{0} \in \Omega_{T}$.

Because $\lambda_{0} \in \varrho_{T}(\dot{x})$, there is a neighbourhood $D_{1}$ of $\lambda_{0}$ and an analytic function $\tilde{f}: D_{1} \rightarrow \dot{X}$ such that $(\lambda \dot{I}-\dot{T}) \tilde{f}(\lambda)=\dot{x}$ on $D_{1}$. We can write $\tilde{f}(\lambda)=\sum_{n=0}^{\infty} \dot{a}_{n}\left(\lambda-\lambda_{0}\right)^{n}$
for every $\lambda \in D_{1}$. We have to find elements $b_{n} \in \dot{a}_{n}(n \geqq 0)$ such that the analytic function $f(\lambda)=$ $=\sum_{n=0}^{\infty} b_{n}\left(\lambda-\lambda_{0}\right)^{n}$ verifies $(\lambda I-T) f(\lambda)=x$.

From

$$
\begin{gathered}
x=(\lambda \dot{I}-\dot{T}) \tilde{j}(\lambda)=(\lambda \dot{I}-\dot{T}) \sum_{n=0}^{\infty} \dot{a}_{n}\left(\lambda-\lambda_{0}\right)^{n}= \\
=\left(\lambda_{0} \dot{a}_{0}-\dot{T} \dot{a}_{0}\right)+\sum_{n=1}^{\infty}\left(\dot{a}_{n-1}+\lambda_{0} \dot{a}_{n}-\dot{T} \dot{a}_{n}\right)\left(\lambda-\lambda_{0}\right)^{n}
\end{gathered}
$$

and from the fact that $\lambda_{0} \in \Omega_{T}$, it follows that

$$
\left(\lambda_{0} \dot{I}-\dot{T}\right) \dot{a}_{0}=\dot{x} \quad \text { and } \quad\left(\lambda_{0} \dot{I}-\dot{T}\right) \dot{a}_{n}=-\dot{a}_{n-1} \quad(n \geqq 1) \text {. }
$$

The first equality implies

$$
\left(\lambda_{0} I-T\right) a_{0}=x+y
$$

with $y \in Y$. Now we use the hypothesis $\lambda_{0} \in \varrho(T \mid Y)$. For $y \in Y$ there is $\alpha_{0} \in Y$ with $\left(\lambda_{0} I-T\right) \alpha_{0}=y$. It follows from (1) that

$$
\left(\lambda_{0} I-T\right)\left(a_{0}-\alpha_{0}\right)=x \text { and } b_{0}=a_{0}-\alpha_{0}, \quad \alpha_{0} \in Y .
$$

In the same way we obtain $b_{l i} \in \dot{a}_{n}$ such that $\left(\lambda_{0} I-T\right) b_{n}=-b_{n-1}$ and thus the analytic function $f$ which verifies the required equality. Hence $\lambda_{0} \in \varrho_{T}(x)$.

$3^{\circ}$ Follows from $1^{\circ}$ and $2^{\circ}$.

Corollary 2.33. Let $T \in \mathscr{P}(X)$, let $Y$ be an invariant subspace for $T$ and $x \in X \backslash Y$. If $Y$ is analyticall. invariant and $\sigma(T \mid Y) \subset \sigma_{T}(\dot{x})$, then

$$
\sigma_{T}(\dot{x})=\sigma_{T}(x) \text {. }
$$

Proof. By the first hypothesis, $\dot{T}$ has the s.v.e.p.. Now, it is easy to prove that $\sigma_{\dot{T}}(\dot{x}) \subset \sigma_{T}(x)$ (even without using the second condition).

Remark 2.34. It is known ([6], Prop. 2.8, 2.1 and 1.15) that, for $T \in \mathscr{P}(X)$ and $Y$ analytically invariant for $T, \sigma(T \mid Y) \subset \sigma(T)$ and $\sigma(\dot{T}) \subset \sigma(T)$. The hypothesis in Corollary 2.33 implies that $\sigma(T \mid Y) \subset \sigma(\dot{T})$.

We give three examples related to Proposition 2.32 and Corollary 2.33. In the first one, the second condition of Corollary 2.33 is not verified and we construct an element $x$ which verifies the equality $\sigma_{T}(\dot{x})=\sigma_{T}(x)$ and another which does not verify this equality. In the second example, the second condition of Corollary 2.33 can 
be fulfilled or it can fail. In the third, the condition $3^{\circ}$ of Proposition 2.32 is always verified and the equality $\sigma_{T}(\dot{x})=\sigma_{T}(x)$ is obvious.

Examples. 1) We use Example B. Let $f \in \mathscr{B}(K) \backslash \mathscr{C}(K)$. In $\overline{\mathscr{B}(K)}=\mathscr{B}(K) / \mathscr{C}(K)$ we denote by $\dot{f}$ the class of $f$.

Denote $D_{f}=\{x \in K \mid f$ is discontinuous in $x\}$.

We observe that $D_{f}$ is invariant for classes (that is, for every $g \in \dot{f}, D_{g}=D_{f}$ ); the converse is not true: one takes $f_{1}$ and $f_{2}$ as below, which are not in the same class, but have the same set of discontinuity.

We show that $\sigma_{\dot{M}_{x}}(\dot{f})=\bar{D}_{f}$ (see Appendix B, 5-6). On the other hand, we prove (Appendix B, 3) that $\sigma_{M_{x}}(f)=\operatorname{Supp} f$.

Let $x_{0} \in K$ and

Hence

$$
\begin{aligned}
& f_{1}\left(x_{0}\right)=\chi_{\left\{x_{0}\right\}}(x), \\
& f_{2}(x)=\left\{\begin{array}{lll}
1 & \text { for } & x=x_{0} \\
2 & \text { for } & x \neq x_{0}
\end{array}\right.
\end{aligned}
$$

$$
\begin{aligned}
& \sigma_{\dot{M}_{x}}\left(\dot{f}_{1}\right)=\sigma_{M_{x}}\left(f_{1}\right)=\left\{x_{0}\right\}, \\
& \sigma_{\dot{M}_{x}}\left(\dot{f}_{2}\right)=\left\{x_{0}\right\}, \text { but } \sigma_{M_{x}}\left(f_{2}\right)=K .
\end{aligned}
$$

We remark that $\sigma\left(M_{x} \mid \mathscr{C}(K)\right) \notin \sigma_{\dot{M}_{x}}\left(\dot{f}_{i}\right)(i=1,2)$.

2) Consider again Example $\mathrm{B}-$ the multiplication by $x$ on $\mathscr{B}(K)$ but with another subspace. Let $F$ be closed in $\mathbb{C}$ and

$$
Y_{F}=\{f \in \mathscr{C}(K) \mid \operatorname{Supp} f \subset F\} .
$$

Denote by $\dot{M}_{x}^{F}$ the co-induced operator of $M_{x}$ on $\mathscr{B}(K) / Y_{F}$.

We show (Appendix B, 7-9) that $Y_{F}$ is analytically invariant for $M_{x}$ (hence $\dot{M}_{x}^{F}$ has the s.v.e.p.) and that $\sigma\left(M_{x} \mid Y_{F}\right)=\overline{\operatorname{Int} F}$. It is easy to prove that $\sigma\left(\dot{M}_{x}^{F}\right)=K$.

For every $f \in \mathscr{B}(K) \backslash Y_{F}$, denote $E=K \backslash$ Int $F$ and $\operatorname{Supp}_{E} f=\overline{\{x \in E \mid f(x) \neq 0\}}$.

We have

(see Appendix B, 10).

$$
\sigma_{M_{x} F}\left(\dot{f}^{F}\right)=\bar{D}_{f} \cup \operatorname{Supp}_{E} f
$$

If we choose $f \in \mathscr{B}(K) \backslash Y_{F}$ such that $D_{f} \supset$ Int $F$, then the second condition of Corollary 2.33 is fulfilled. It is easy to prove that, in this case, $\bar{D}_{f} \cup \operatorname{Supp}_{E} f=\operatorname{Supp} f$ (that is $\sigma_{\dot{M}_{x}} \boldsymbol{F}\left(f^{F}\right)=\sigma_{M_{x}}(f)$ ).

On the other hand, if we consider

$$
f_{0}(x)=\left\{\begin{array}{ll}
1 & \text { for } x=x_{0}, \\
2 & \text { for } x \neq x_{0}
\end{array},\right.
$$

with $x_{0} \notin F$, then we have $\sigma_{M_{x} F}\left(\dot{f}_{0}^{F}\right)=K \backslash$ Int $F$ and $\sigma_{M_{x}}(f)=K$; note that the second condition of the Corollary is not fulfilled.

3) Consider Example A. Denote $D_{1}=\{\lambda \in \mathbb{C}|| \lambda \mid \leqq 1\}$.

We know that $\sigma_{U^{*}}(x)=D_{1}$ for every $x \in l^{2}$. It is easy to prove (Appendix A, 3) 
that $\sigma\left(U^{*} \mid E_{\lambda}\right)=\{\lambda\}, S_{\dot{U}^{*}}=D_{1}$ and $\sigma\left(\dot{U}^{*}\right)=D_{1}$, where $\dot{U}^{*}$ denotes the co-induced operator of $U^{*}$ on $l^{2} / E_{\lambda}$. Therefore $\sigma_{\dot{U}}(\dot{x})=D_{1}$ for every $\dot{x} \in l^{2} / E_{\lambda}$.

It follows that the condition $3^{\circ}$ of Proposition 2.32 is fulfilled. The equality of the local spectra is obvious.

d) Properties of permanence for the weak spectral equivalence. Using the results obtained in c), we can give now some conditions under which the weak spectral equivalence is preserved by passing to subspaces or quotient spaces.

Proposition 2.35. Let $T_{i} \in \mathscr{L}(X)$ and let $Y$ be an invariant subspace for $T_{i}(i=1,2)$. Suppose that $Y$ is $\Omega_{T_{i}}$ analytically invariant for $T_{i}$ and $\left.\Omega_{T_{i}}\right|_{Y}=\Omega_{T_{i}}$ for $i=1,2$.

If $T_{1} \sim{ }^{w} T_{2}$, then $T_{1}\left|Y \sim{ }^{w} T_{2}\right| Y$.

Proof. Apply Proposition 2.27.

Proposition 2.36. Let $T_{i} \in \mathscr{L}(X)$, let $Y$ be an invariant subspace for $T_{1}(i=1,2)$. Suppose that

$1^{\circ} S_{T_{i} \mid Y}=S_{T_{i}} \cap \sigma\left(T_{i} \mid Y\right)(i=1,2)$,

and for every $x \in Y$,

$\left.\begin{array}{ll}2^{\circ} & \gamma_{T_{i}}(x) \gamma_{T_{i} \mid Y}(x) \\ 3^{\circ} & \sigma_{T_{i}}(x) \subset \sigma\left(T_{i} \mid Y\right) \cup \gamma_{T_{i}}(x),\end{array}\right\}(i=1,2)$.

If $T_{1} \sim{ }^{w} T_{2}$, then $T_{1}\left|Y \sim{ }^{w} T_{2}\right| Y$.

Proof. Apply Proposition 2.29.

Corollary 2.37. Let $T_{i} \in \mathscr{P}(X)$ and let $Y$ be an analytically invariant subspace for $T_{i}(i=1,2)$. If $T_{1} \sim^{w} T_{2}$, then $T_{1}\left|Y \sim^{w} T_{2}\right| Y$.

Proposition 2.38. Let $T_{i} \in \mathscr{L}(X)$, let $Y$ be an invariant subspace for $T_{i}(i=1,2)$. Suppose that, for every $x \in X \backslash Y, \sigma\left(T_{i} \mid Y\right) \subset \sigma_{T_{i}}(x) \cap \sigma_{T_{i}}(\dot{x})$.

If $T_{1} \sim{ }^{w} T_{2}$, then $\dot{T}_{1} \sim{ }^{w} \dot{T}_{2}$.

Proof. For $x \in X \backslash Y, \sigma_{T_{1}}(\dot{x})=\sigma_{T_{2}}(\dot{x})$ by Proposition 2.32; obviously for $x \in Y$, $\sigma_{T_{1}}(\dot{x})=\sigma_{T_{2}}(\dot{x})=S_{T_{1}}=S_{T_{2}}$ by Proposition 2.11, b).

Corollary 2.39. Let $T_{i} \in \mathscr{P}(X)$, let $Y$ be an analytically invariant subspace for $T_{i}(i=1,2)$. Suppose that for every $x \in X \backslash Y, \sigma\left(T_{i} \mid Y\right) \subset \sigma_{T_{i}}(\dot{x})(i=1,2)$.

If $T_{1} \sim{ }^{w} T_{2}$, then $\dot{T}_{1} \sim{ }^{w} \dot{T}_{2}$.

\section{WEAK SPECTRAL CONVERGENCE}

a) The semi-metric $p_{w}$. In 2, a) the semi-metric $p_{w}$ was defined and some of its properties were established, while in 1 , a) was defined $p(A, B)$ for two sets $A, B \subset \mathbb{C}$. Restricted to nonvoid compact sets, $p$ is a metric (the Hasudorff metric).

Consider now $\mathscr{L}(X)$ with the topology generated by $p_{w}$ and $\mathscr{K}=\{K \subset \mathbb{C} \mid K$ com- 
pact $\}$ with the topology given by the metric $p$. With respect to these topologies, we have

Proposition 3.1. a) The map $\Sigma: \mathscr{L}(X) \rightarrow \mathscr{K}$ defined by $\Sigma(T)=\sigma(T)$ is a contraction.

b) For every $x \in X$, the map $\Sigma_{x}: \mathscr{L}(X) \rightarrow \mathscr{K}$ defined by $\Sigma_{x}(T)=\sigma_{T}(x)$ is a contraction.

Proof. a) We use Lemma 1.4:

$$
\begin{gathered}
\delta\left(\sigma\left(T_{1}\right), \sigma\left(T_{2}\right)\right)=\delta\left(\bigcup_{x \in X} \sigma_{T_{1}}(x), \bigcup_{y \in X} \sigma_{T_{2}}(y)\right)= \\
=\sup _{x \in X} \inf \delta\left(\sigma_{T_{1}}(x), \sigma_{T_{2}}(y)\right) \leqq \sup _{x \in X} \delta\left(\sigma_{T_{1}}(x), \sigma_{T_{2}}(x)\right)=\delta_{w}\left(T_{1}, T_{2}\right),
\end{gathered}
$$

therefore $p\left(\sigma\left(T_{1}\right), \sigma\left(T_{2}\right) \leqq p_{w}\left(T_{1}, T_{2}\right)\right.$ by symmetry.

b) For a fixed $x$, obviously

$$
\delta\left(\sigma_{T_{1}}(x), \sigma_{T_{2}}(x)\right) \leqq \sup _{x \in X} \delta\left(\sigma_{T_{1}}(x), \sigma_{T_{2}}(x)\right)=\delta_{w}\left(T_{1}, T_{2}\right)
$$

so that $p\left(\sigma_{T_{1}}(x), \sigma_{T_{2}}(x)\right) \leqq p_{w}\left(T_{1}, T_{2}\right)$.

Remark 3.2. With the notation from Sec. 1, for $T_{1}, T_{2} \in \mathscr{P}(X)$, the following inequalities hold:

$$
\begin{aligned}
& p\left(\sigma\left(T_{1}\right), \sigma\left(T_{2}\right)\right) \leqq p_{w}\left(T_{1}, T_{2}\right) \leqq p\left(T_{1}, T_{2}\right), \\
& p\left(\sigma_{T_{1}}(x), \sigma_{T_{2}}(x)\right) \leqq p_{w}\left(T_{1}, T_{2}\right) \leqq p\left(T_{1}, T_{2}\right) .
\end{aligned}
$$

The inequalities between the first and the third terms are known ([1], II, Prop. 1.4 and 1.7).

For non-void $A$ in $\mathbb{C}$ and $r>0$ denote

$$
C(A, r)=\{\lambda \in \mathbb{C} \mid \operatorname{dist}(A, \lambda) \leqq r\} .
$$

Lemma 3.3. Let $T_{1}, T_{2} \in \mathscr{L}(X)$ with $p_{w}\left(T_{1}, T_{2}\right)<\varepsilon$. For $i, j=1,2, i \neq j$,

a) $\sigma_{T_{i}}(x) \subset C\left(\sigma_{T_{j}}(x), \varepsilon\right)(\forall) x \in X$,

b) $X_{T_{i}}(F) \subset X_{T_{j}}(C(F, \varepsilon))(\forall) F \subset \mathbb{C}$ closed.

Proof. a) is obvious from the definition of $p_{w}$.

b) Let $x \in X_{T_{1}}(F)$, that is, $\sigma_{T_{1}}(x) \subset F$. We need $x \in X_{T_{2}}\left(C(F, \varepsilon)\right.$, that is, $\sigma_{T_{2}}(x) \subset$ с $C(F, \varepsilon)$.

Let $\lambda \in \sigma_{T_{2}}(x) \subset C\left(\sigma_{T_{1}}(x), \varepsilon\right)$ (by a)); $\sigma_{T_{1}}(x)$ being compact, there is $\mu \in \sigma_{T_{1}}(x)$ with $|\lambda-\mu| \leqq \varepsilon$. But $\sigma_{T_{2}}(x) \subset F$. Thus we obtain, for $\lambda \in \sigma_{T_{2}}(x)$, a $\mu \in F$ with $|\lambda-\mu| \leqq \varepsilon$. It follows that $\sigma_{T_{2}}(x) \subset C(F, \varepsilon)$.

b) Weak spectral convergence. Definition 3.4. Let $T_{n}, T_{0} \in \mathscr{L}(X)$. We say that 
the sequence $\left\{T_{n}\right\}$ converges weakly spectrally to $T_{0}$ if

$$
\lim _{n \rightarrow \infty} p_{w}\left(T_{n}, T_{0}\right)=0 .
$$

We denote this by $T_{n} \rightarrow^{w-\text { sp }} T_{0}$

Remark 3.5. Note that the weak spectral limit is not unique, but any two limits of a sequence are weakly equivalent.

Remark 3.6. If $T_{n}, T_{0} \in \mathscr{P}(X)$, it is obvious that the spectral convergence (see Definition 1.11) implies the weak spectral convergence. This is not true if $T_{n} \notin \mathscr{P}(X)$.

Example. Let $U^{*} \in \mathscr{L}\left(l^{2}\right)$ be the adjoint of the unilateral shift and $T_{n}=(1 / n) U^{*}$. In this case $T_{n} \rightarrow^{\text {sp }} 0$, but $T_{n} \rightarrow^{w-\text { sp }} 0$.

Indeed,

$$
d_{\mathrm{sp}}\left(0, T_{n}\right)=d_{\mathrm{sp}}\left(T_{n}, 0\right)=\varlimsup_{m \rightarrow \infty}\left\|\left(T_{n} \backslash 0\right)^{[m]}\right\|^{1 / m}=\varlimsup_{m \rightarrow \infty} \frac{1}{n}\left\|\left(U^{*}\right)^{m}\right\|^{1 / m}=\frac{1}{n}\left\|U^{*}\right\|_{\mathrm{sp}}=\frac{1}{n},
$$

therefore it converges to zero.

On the other hand, $\delta_{w}\left(T_{n}, 0\right)=\sup _{x \in X} \delta\left(\sigma_{T_{n}}(x), \sigma_{0}(x)\right)$. For $x=0, \quad \sigma_{T_{n}}(0)=$ $=\sigma_{(1 / n) U^{*}}(0)=(1 / n) o_{U^{*}}(0)=(1 / n) D_{1}=D_{(1 / n)}$ and $D_{(1 / n)}$ is not void $\left(D_{(1 / n)}=\right.$ $=\{\lambda|| \lambda \mid \leqq 1 / n\})$, but $\sigma_{0}(0)=\emptyset$, because $0 \in \mathscr{P}(X)$.

We obtain $\delta\left(\sigma_{T_{n}}(0), \sigma_{0}(0)\right)=\delta\left(D_{(1 / n)}, \emptyset\right)=\infty$ for every $n$, therefore $\delta_{w}\left(T_{n}, 0\right)$ and $p_{w}\left(T_{n}, 0\right)$ are infinite. Hence $p_{w}\left(T_{n}, 0\right) \rightarrow 0$.

Proposition 3.7. If $T_{n}, T_{0} \in \mathscr{L}(X)$ and $T_{n} \rightarrow^{w-\text { sp }} T_{0}$, then

$$
\lim _{n \rightarrow \infty} p\left(\sigma\left(T_{n}\right), \sigma(T)\right)=0 \text {. }
$$

Proof. This is a consequence of Proposition 3.1, a).

Theorem 3.8. Let $T_{n}, T_{0} \in \mathscr{L}(X)$ with $T_{n} \rightarrow^{w-s p} T_{0}$. If all $T_{n}(n \geqq 1)$ have the s.v.e.p., then $T_{0}$ has the s.v.e.p. as well.

Proof. Let $\varepsilon>0$ and $n \geqq n_{\varepsilon}$ such that $p_{w}\left(T_{n} ; T_{0}\right)<\varepsilon$. We have $\delta_{w}\left(T_{0}, T_{n}\right)=$ $=\sup _{x \in X} \delta\left(\sigma_{T_{0}}(x), \sigma_{T_{n}}(x)\right)<\varepsilon$, so that for every $x, \delta\left(\sigma_{T_{0}}(x), \sigma_{T_{n}}(x)<\varepsilon\right.$. In particular for $x=0: \delta\left(S_{T_{0}}, S_{T_{n}}\right)<\varepsilon$. By hypothesis, $S_{T_{n}}=\emptyset$. If we suppose $S_{T_{0}} \neq \emptyset$, then $\delta\left(S_{T_{0}}, \emptyset\right)=\infty \nless \varepsilon$. Therefore $S_{T_{0}}=\emptyset$.

Theorem 3.9. Let $T_{n}, T_{0} \in \mathscr{L}(X)$ with $T_{n} \rightarrow^{w-\text { sp }} T_{0}$. If all $T_{n}(n \geqq 1)$ are decomposable, then so is $T_{0}$.

Proof. $T_{0}$ has the s.v.e.p. by Theorem 3.8 .

Now the proof is similar to that in [2], Th. 2.7, using Lemma 3.3 instead of [2], Cor. 2.5 and Proposition 3.1 instead of [2], Prop. 2.2. 
Remark 3.10. The previous Example shows that $\mathscr{L}(X)$ is not complete in the $p_{w}$-topology.

Indeed,

$$
p_{w}\left(T_{n}, T_{m}\right)=\left|\frac{1}{n}-\frac{1}{m}\right| \rightarrow 0
$$

for $m, n \rightarrow \infty$. Suppose that $p_{w}\left(T_{n}, T_{0}\right) \rightarrow 0$. By Proposition 3.7, $\lim p\left(\sigma\left(T_{n}\right), \sigma\left(T_{0}\right)\right)=$ $=0$. But $\sigma\left(T_{n}\right)=D_{(1 / n)}$ and $\lim _{n} p\left(D_{(1 / n)},\{0\}\right)=0$. The limit in ${ }^{n} \mathscr{K}$ being unique, it follows that $\sigma\left(T_{0}\right)=\{0\}$ and hence $S_{T_{0}}=\emptyset$. But this is impossible (the proof is the same as in the previous example).

\section{APPENDIX}

Some of the results (A1, B3) are known, but we give them for the sake of completeness.

\section{A. THE ADJOINT OF THE UNILATERAL SHIFT}

Let $l^{2}$ be the space of square-summable sequences (of complex numbers) and $U \in$ $\in \mathscr{L}\left(l^{2}\right)$ the unilateral shift on $l^{2}$, given by

$$
U\left(\xi_{0}, \xi_{1}, \xi_{2}, \ldots\right)=\left(0, \xi_{0}, \xi_{1}, \ldots\right), \quad\left\{\xi_{n}\right\}_{n} \in l^{2} .
$$

Let $U^{*}$ be the adjoint of $U$. Hence

$$
U^{*}\left(\xi_{0}, \xi_{1}, \xi_{2}, \ldots\right)=\left(\xi_{1}, \xi_{2}, \xi_{3}, \ldots\right),\left\{\xi_{n}\right\}_{n} \in l^{2}
$$

1. $U^{*}$ has not the s.v.e.p. Let $\omega \subset\{\lambda|| \lambda \mid>1\}$ be an open set and $f: \omega \rightarrow l^{2}$ an analytic function such that $\left(\lambda I-U^{*}\right) f(\lambda) \equiv 0$ on $\omega$; that is $\left(\lambda I-U^{*}\right)\left\{f_{n}(\lambda)\right\}_{n \geqq 0} \equiv$ $\equiv 0$ on $\omega$. We obtain $\lambda f_{n}(\lambda)-f_{n+1}(\lambda)=0$, so that $f_{n}(\lambda)=\lambda^{n} f_{0}(\lambda)$. Suppose $f_{0}(\lambda) \neq 0$.

We have $\left\{f_{n}(\lambda)\right\}_{n \geqq 0} \in l^{2}$, that is the series $\sum_{n=0}^{\infty}|\lambda|^{2 n}\left|f_{0}(\lambda)\right|^{2}=\left|f_{0}(\lambda)\right|^{2} \sum_{n=0}^{\infty}|\lambda|^{2 n}$ must be convergent, which is impossible, because $|\lambda|>1$. We conclude that $f_{0}(\lambda)=0$, hence $f_{n}(\lambda)=0(\forall) n$.

Hence $\omega \subset\{\lambda|| \lambda \mid>1\}$ implies that $\omega \subset \Omega_{U^{*}}$, so that $\{\lambda|| \lambda \mid>1\} \subset \Omega_{U^{*}}$.

Now, for every $\lambda \neq 0$ with $|\lambda|<1$ we have $\left(\lambda I-U^{*}\right)\left\{\lambda^{n}\right\}_{n \geqq 0}=0$ and the function $\lambda \rightarrow\left\{\lambda^{n}\right\}_{n \geqq 0}$ is analytic and $\neq 0$. Hence $\lambda$ does not belong to any open set of analytic uniqueness $\omega_{i} \lambda \in \bigcap \subset \omega=\complement \bigcup \omega=C \Omega_{U^{*}}=S_{U^{*}}{ }^{*}$.

Therefore $\{\lambda|0<| \lambda \mid<1\} \subset S_{U^{*}}$, so that by passing to the closure, $\{\lambda|| \lambda \mid \leqq 1\} \subset S_{U^{*}}$, or $\{\lambda|| \lambda \mid>1\} \subset \Omega_{U^{*}}$. Hence $\Omega_{U^{*}}=\{\lambda|| \lambda \mid>1\}$ and $S_{U^{*}}=\{\lambda \in \mathbb{C}|| \lambda \mid \leqq 1\}$.

We have denoted by $E_{\lambda_{0}}$ the eigenspace corresponding to $\lambda_{0}\left(0<\left|\lambda_{0}\right|<\frac{1}{2}\right)$. 
2. $E_{\lambda_{0}}$ is not a spectral maximal space for $U^{*}$. Define, for $\lambda_{0} \in \mathbb{C} \backslash\{0\}$ with $\left|\lambda_{0}\right|<\frac{1}{2}, t_{0}=\lambda_{0}, t_{n+1}=\lambda_{0} t_{n}-\lambda_{0}^{n}$ and

$$
Z=\left\{r\left\{\lambda_{0}^{n}\right\}_{n \geqq 0}+\alpha\left\{t_{n}\right\}_{n \geqq 0} \mid r, \alpha \in \mathbb{C}\right\} .
$$

a) $Z$ is invariant under $U^{*}$.

Let $Z=\left\{r \lambda_{0}^{n}+\alpha t_{n}\right\}_{n \geqq 0} \in Z$.

$$
\begin{gathered}
U^{*}(z)=\left\{r \lambda_{0}^{n+1}+\alpha t_{n+1}\right\}_{n \geqq 0}=\left\{r \lambda_{0}^{n+1}+\alpha \lambda_{0} t_{n}-\alpha \lambda_{0}^{n}\right\}_{n \geqq 0}= \\
=\left\{\left(r \lambda_{0}-\alpha\right) \lambda_{0}^{n}+\left(\alpha \lambda_{0}\right) t_{n}\right\}_{n \geqq 0} \in Z .
\end{gathered}
$$

b) $Z \stackrel{\supsetneq}{\ddagger} E_{\lambda_{0}}$ obviously.

c) $\sigma\left\{U^{*} \mid Z\right)=\left\{\lambda_{0}\right\}$, that is, for every $\lambda \neq \lambda_{0},\left(\lambda I-U^{*} \mid Z\right)$ is invertible.

a) Let $z \in Z$ with $\left(\lambda I-U^{*}\right) z=0$.

Note that $t_{n}=\lambda_{0}^{n+1}-n \lambda_{0}^{n+1}, z=\left\{z_{n}\right\}_{n \geqq 0}$ with $z_{0}=r+\alpha \lambda_{0}, z_{n}=r \lambda_{0}^{n}+\alpha \lambda_{0}^{n+1}-$ $-\alpha n \lambda_{0}^{n-1}(n \geqq 1)$ and $\left(\lambda I-U^{*}\right) z=0$.

Therefore, for every $n \geqq 1$,

$$
\begin{gathered}
\lambda r \lambda_{0}^{n}-r \lambda_{0}^{n+1}=\alpha\left(-\lambda \lambda_{0}^{n+1}+\lambda_{0}^{n+2}-n \lambda_{0}^{n}+n \lambda \lambda_{0}^{n-1}\right), \\
r \lambda_{0}^{n}\left(\lambda-\lambda_{0}\right)=\alpha \lambda_{0}^{n-1}\left(\lambda-\lambda_{0}\right)\left(-\lambda_{0}^{2}+n\right) .
\end{gathered}
$$

We divide by $\lambda_{0}^{n-1}\left(\lambda-\lambda_{0}\right) \neq 0$ and obtain $r \lambda_{0}=\alpha\left(n-\lambda_{0}^{2}\right)$.

If we suppose $\alpha \neq 0$, letting $n \rightarrow \infty$, we obtain a contradiction. Therefore $\alpha=0$, so that $r=0$. It follows that $z=0$.

Hence $\left(\lambda I-U^{*} \mid Z\right)$ is injective.

$\beta)$ Let $y=\left\{r_{0} \lambda_{0}^{n}+\alpha_{0} t_{n}\right\}_{n}$. Let us find $r$ and $\alpha$ in $\mathbb{C}$ such that $\left(\lambda I-U^{*}\right) z=y$ where $z=\left\{r \lambda_{0}^{n}+\alpha t_{n}\right\}_{n \geqq 0}$.

Take

$$
\alpha=\frac{\alpha_{0}}{\lambda-\lambda_{0}} \quad \text { and } \quad r=\frac{r_{0}}{\lambda-\lambda_{0}}-\frac{\alpha_{0}}{\left(\lambda-\lambda_{0}\right)^{2}}
$$

Therefore

$$
z=\left\{\frac{r_{0} \lambda_{0}^{n}}{\lambda-\lambda_{0}}-\frac{\alpha_{0} \lambda_{0}^{n}}{\left(\lambda-\lambda_{0}\right)^{2}}+\frac{\alpha_{0} t_{n}}{\lambda-\lambda_{0}}\right\}_{n \geqq 0}
$$

and $\left(\lambda I-U^{*}\right) z=\left\{r_{0} \lambda_{0}^{n}+\alpha_{0} t_{n}\right\}_{n \geqq 0}=y$.

Hence $\left(\lambda I-U^{*} \mid Z\right)$ is also surjective.

3. a) It is obvious that $\sigma\left(U^{*} \mid E_{\lambda}\right)=\{\lambda\}$.

b) We know that $\sigma\left(U^{*}\right) \subset \sigma\left(\dot{U}^{*}\right) \cup \sigma\left(U^{*} \mid E_{\lambda}\right)$ and $\sigma\left(\dot{U}^{*}\right) \subset \sigma\left(U^{*}\right) \cup \sigma\left(U^{*} \mid E_{\lambda}\right)$ (see, for instance, [6], Prop. 1.14). Hence $D_{1} \subset \sigma\left(\dot{U}^{*}\right) \cup\{\lambda\} \subset D_{1}$, that is $\sigma\left(\dot{U}^{*}\right) \cup$ $\cup\{\lambda\}=D_{1}$, so that $\sigma\left(\dot{U}^{*}\right)=D_{1}\left(\sigma\left(\dot{U}^{*}\right)\right.$ being closed, it cannot equal $\left.D_{1} \backslash\{\lambda\}\right)$.

c) $\dot{U}^{*}$ has not the s.v.e.p., because $E_{\lambda}$ is not analytically invariant for $U^{*}$. Hence $S_{U^{*}} \neq \emptyset$. It is known (see, for instance, [3], Prop. 1.1.1) that $S_{U^{*}} \subset S_{U^{*}} \cup \sigma\left(U^{*} \mid E_{\lambda}\right)$ and $S_{U^{*}} \subset S_{\dot{U}^{*}} \cup \sigma\left(U^{*} \mid E_{\lambda}\right)$. Therefore $S_{U^{*}} \subset D_{1}$ and $D_{1} \subset S_{\dot{U}^{*}} \cup\{\lambda\}$, so that $S_{U^{*}} \cup\{\lambda\}=D_{1}$ and we conclude in the same way that $S_{U^{*}}=D_{1}$. 
Let $K$ be compact in $\mathbb{C}, \mathscr{B}(K)=\{f: K \rightarrow \mathbb{C} \mid f$ bounded $\}$ with the sup-norm denoted by \|\|$_{0}$, and $\mathscr{C}(K)=\{f: K \rightarrow \mathbb{C} \mid f$ continuous $\}$.

Let $M_{x} \in \mathscr{L}(\mathscr{B}(K))$ be defined by $M_{x} f(x)=x f(x)(\forall) x \in K$.

It is known that $\sigma\left(M_{x}\right)=K$.

1. $M_{x}$ has the s.v.e.p. Let $D$ be an open connected set in $\mathbb{C}$ and let $h: D \rightarrow \mathscr{B}(K)$ be an analytic function with $\left(\lambda I-M_{x}\right) h_{\lambda} \equiv 0$ for every $\lambda \in D$, that is $(\lambda-x) h_{\lambda}(x)=$ $=0$ for every $\lambda \in D$ and $x \in K$.

Let $\omega=D \cap C K$; it is an open set. Suppose that it is not void. For $\lambda \in \omega, x \in K$ we have $\lambda-x \neq 0$, so that $h_{\lambda}(x)=0$ for every $x \in K$, that is $h_{\lambda}=0$ for every $\lambda \in \omega$; because $h$ is analytic and $\omega$ is open in $D$, it follows that $h \equiv 0$ on $D$.

If $\omega=\emptyset$, then $D \subset K$. Fix $\lambda_{0} \in D$. We have $\left(\lambda_{0}-x\right) h_{\lambda_{0}}(x) \equiv 0$. For $x \neq \lambda_{0}$ we have $h_{\lambda_{0}}(x)=0$, so that $h_{\lambda_{0}}(x)=\alpha_{\lambda_{0}} \chi_{\left\{\lambda_{0}\right\}}(x)$. Note that we can take $\alpha_{\lambda_{0}} \equiv 1$. We show that such a function cannot be analytic with respect to $\lambda$ (it is not continuous).

Let $0<\varepsilon<1$ and let $\lambda$ fulfil $\left|\lambda-\lambda_{0}\right|<\varepsilon$. Therefore

$$
\begin{gathered}
\left\|h_{\lambda_{0}}-h_{\lambda}\right\|_{0}=\sup _{x \in K}\left|h_{\lambda_{0}}(x)-h_{\lambda}(x)\right|=\sup _{x \in K}\left|\chi_{\left\{\lambda_{0}\right\}}(x)-\chi_{\{\lambda\}}(x)\right|= \\
=\sup _{x \in K}\left\{\begin{array}{lll}
0 & \text { for } x \neq \lambda, \quad x \neq \lambda_{0} \\
1 & \text { for } x=\lambda \text { or } x=\lambda_{0}
\end{array}=1 \nless \varepsilon .\right.
\end{gathered}
$$

2. $\mathscr{C}(K)$ is analytically invariant for $M_{x}$. Let $\omega$ be an open set in $\mathbb{C}$ and $g: \omega \rightarrow$ $\rightarrow \mathscr{B}(K)$ an analytic function with $\left(\lambda I-M_{x}\right) g_{\lambda} \in \mathscr{C}(K)$. Therefore $\left(\lambda I-M_{x}\right) g_{\lambda}=$ $=f_{\lambda}$ with $f: \omega \rightarrow \mathscr{C}(K)$ analytic $\left(f_{\lambda}(x)=(\lambda-x) g_{\lambda}(x)\right.$ and $g_{\lambda}$ is analytic $)$. We have to prove that $g_{\lambda} \in \mathscr{C}(K)$.

For every $x \neq \lambda, g_{\lambda}(x)=f_{\lambda}(x) /(\lambda-x)$, hence $g_{\lambda}$ is continuous on $K \backslash\{\lambda\}$. From the definition of $f_{\lambda}$ we have $f_{\lambda}(\lambda)=0(\lambda \leqq \omega \cap K)$.

Because $f_{\lambda}: \omega \rightarrow \mathscr{C}(K)$ is an analytic function, we have $f_{\lambda}=\sum_{n=0}^{\infty} a_{x, n}(\lambda-x)^{n}$ with $a_{x, n} \in \mathscr{C}(K), a_{x, 0}=f_{x}$, the series being convergent whenever $|\lambda-x|<$ $<\operatorname{dist}(x, \operatorname{Fr} \omega)$.

Therefore $f_{\lambda}(x)=\sum_{n=0}^{\infty} a_{x, n}(x)(\lambda-x)^{n}=\sum_{n=1}^{\infty} a_{x, n}(x)(\lambda-x)^{n} \quad$ (because $a_{x, 0}(x)=$
$\left.=f_{x}(x)=0\right)$ and

$$
\frac{f_{\lambda}(x)}{\lambda-x}=\sum_{n=1}^{\infty} a_{x, n}(x)(\lambda-x)^{n-1}
$$

hence $\lim _{\lambda \rightarrow x} f_{\lambda}(x) /(\lambda-x)$ exists and is equal to $a_{x, 1}(x)$.

For $\mu \neq \lambda, \mu$ in the domain of convergence of the series, we have

$$
g_{\mu}(\lambda)=\frac{f_{\mu}(\lambda)}{\mu-\lambda} .
$$


Hence there exists

$$
\lim _{\mu \rightarrow \lambda} g_{\mu}(\lambda)=\lim _{\mu \rightarrow \lambda} \frac{f_{\mu}(\lambda)}{\mu-\lambda}=a_{\lambda, 1}(\lambda) .
$$

On the other hand, $g_{\lambda}$ is analytic, so that $g_{\lambda}(\lambda)=\lim _{\mu \rightarrow \lambda} g_{\mu}(\lambda)$. Hence $g_{\lambda}(\lambda)=a_{\lambda, 1}(\lambda)$
and $g_{\lambda}$ is continuous also in $\lambda$.

3. For every $f \in \mathscr{B}(K), \sigma_{M_{x}}(f)=\operatorname{Supp} f$. We prove that $\varrho_{M_{x}}(f)=\mathbb{C} \backslash \overline{\{x \mid f(x) \neq 0\}}$.

Let $\lambda_{0} \in \mathbb{C} \backslash \overline{\{x \mid f(x) \neq 0\}}$ : there is a neighbourhood $V_{\lambda_{0}}^{1}$ of $\lambda_{0}$ with the property that $f(x)=0$ for every $x \in K \cap V_{\lambda_{0}}^{1}$. We take $V_{\lambda_{0}}$ open with the property that $\lambda_{0} \in$ $\in V_{\lambda_{0}} \subset \bar{V}_{\lambda_{0}} \subset V_{\lambda_{0}}^{1}$. Denote $d=\operatorname{dist}\left(V_{\lambda_{0}}, C V_{\lambda_{0}}^{1}\right)$. Define $g: V_{\lambda_{0}} \rightarrow \mathscr{B}(K)$ by

$$
g_{\lambda}(x)=\left\{\begin{array}{ccc}
\frac{f(x)}{\lambda-x} & \text { for } & x \neq \lambda \\
0 & \text { for } & x=\lambda .
\end{array}\right.
$$

Note that $g(x)=0$ for every $x \in K \cap V_{\lambda_{0}}^{1}$ (because $\lambda \in V_{\lambda_{0}} \subset V_{\lambda_{0}}^{1}$ ). For $x \in K \cap$ $\cap C V_{\lambda_{0}}^{1}$ we have $\left|g_{\lambda}(x)\right|=|f(x) /(\lambda-x)| \leqq\|f\|_{0} / d$ because $\lambda \in V_{\lambda_{0}}$ and $x \notin V_{\lambda_{0}}^{1}$. Therefore $\left\|g_{\lambda}\right\|_{0} \leqq\|f\|_{0} / d$, that is $g_{\lambda} \in \mathscr{B}(K)$.

It is obvious that $g$ is analytic as a function of $\lambda$ and that $(\lambda-x) g_{\lambda}(x)=f(x)$.

Hence $\mathbb{C} \backslash \operatorname{Supp} f \subset \varrho_{M_{x}}(f)$.

Let now $\lambda \in\{x \mid f(x) \neq 0\}$. Suppose that there exist a neighbourhood $V_{\lambda}$ of $\lambda$ and $g: V_{\lambda} \rightarrow \mathscr{B}(K)$ with $(\lambda-x) g_{\lambda}(x)=f(x)$ for every $x \in K$. For $x=\lambda, f(\lambda) \neq 0$ and $0 \cdot g_{\lambda}(\lambda)=f(\lambda)$. This is a contradiction. Hence $\lambda \in \sigma_{M_{x}}(f)$.

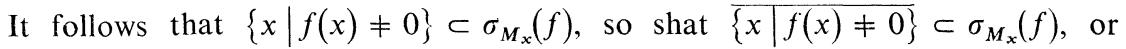
$\varrho_{M_{x}}(f) \subset \mathbb{C} \backslash \overline{\{x \mid f(x) \neq 0\}}$.

4. For every $f \in \mathscr{C}(K), \sigma_{M_{x} \mid \mathscr{C}(K)}(f)=\operatorname{Supp} f$. The proof is the same as above; $g_{\lambda}$ is continuous for $f$ continuous.

We recall that, for $f \in \mathscr{B}(K), D_{f}=\{x \in K \mid f$ is discontinuous in $x\}$ and that we denoted by $\dot{f}$ the class of $f$ in $\mathscr{B}(K) / \mathscr{C}(K)$.

5. For every $g \in \dot{f}, D_{g}=D_{f}$. Suppose that $D_{f} \backslash D_{g} \neq \emptyset$. Let $x \in D_{f} \backslash D_{g}$. We know that $g=f+c(c \in \mathscr{C}(K))$; $c$ and $g$ are continuous in $x$, but $f$ is not. This is a contradiction.

Analogously, $D_{g} \backslash D_{f}=\emptyset$.

6. For every $f \in \mathscr{B}(K) \backslash \mathscr{C}(K), \sigma_{\dot{M}_{x}}(\dot{f})=\bar{D}_{\boldsymbol{f}}$. We show that

$$
\varrho_{M_{x}}(f)=\mathbb{C} \backslash D_{f}=(\mathbb{C} \backslash K) \cup \operatorname{Int}\{x \in K \mid f \text { continuous in } x\} .
$$

Let $\lambda_{0} \in \varrho_{\dot{M}_{x}}(\dot{f})$ : there exist $\omega \rightarrow \lambda_{0}$ and an analytic function $\dot{g}: \omega \rightarrow \mathscr{B}(K) / \mathscr{C}(K)$ with $\left(\lambda \dot{I}-\dot{M}_{x}\right) \dot{g}_{\lambda}=\dot{f}$ for every $\lambda \in \omega$. Therefore

$$
(\lambda-x) g_{\lambda}(x)=f(x)+\varphi_{\lambda}(x)
$$

for every $\lambda \in \omega, x \in K$, where $\varphi_{\lambda} \in \mathscr{C}(K)$ and $\lambda \rightarrow \varphi_{\lambda}$ is analytic. 
On $\omega \cap K$, the function $x \rightarrow \varphi_{x}(x)$ is continuous. This follows immediately from the inequality

$$
\left|\varphi_{x}(x)-\varphi_{x^{\prime}}\left(x^{\prime}\right)\right| \leqq\left|\varphi_{x}(x)-\varphi_{x^{\prime}}(x)\right|+\left|\varphi_{x^{\prime}}(x)-\varphi_{x^{\prime}}\left(x^{\prime}\right)\right|
$$

and from the analyticity of $\varphi_{\lambda}$ (for the first term) and from the continuity of $\varphi$ (for the second).

If $\lambda \in \omega \cap K$, we take $x=\lambda$ in (1); we obtain $f(\lambda)=-\varphi_{\lambda}(\lambda)$, therefore $f$ is continuous in $\lambda$; hence $x \in K \backslash D_{f}$.

If $f \in \omega \backslash K$, then $\lambda \in \mathbb{C} \backslash K$. Therefore $\varrho_{M_{x}}(\dot{f}) \subset \mathbb{C} \backslash D_{j}$.

Let now $\lambda_{0} \in \mathbb{C} \backslash \bar{D}_{f}=(\mathbb{C} \backslash K) \cup$ Int $\{x \in K \mid f$ continuous in $x\}$.

If $\lambda_{0} \in \mathbb{C} \backslash K=\varrho\left(\dot{M}_{x}\right)$, then $\lambda_{0} \in \varrho_{\dot{M}_{x}}(\dot{f})$.

If $\lambda_{0} \in$ Int $\{x \in K \mid f$ continuous in $x\}$, there exists $V_{\lambda_{0}} \subset \bar{V}_{\lambda_{0}} \subset V_{\lambda_{0}}^{1} \subset\{x \mid f$ continuous in $x\}$, so that there exists $V_{\lambda_{0}} \subset \bar{V}_{\lambda_{0}} \subset V_{\lambda_{0}}^{1}$ with $\left.f\right|_{\bar{V}_{\lambda_{0}}}$ continuous.

Define $c: K \rightarrow$ Ccontinuous and such that $\left.c\right|_{\bar{V}_{\lambda_{0}}}=-\left.f\right|_{\bar{V}_{\lambda_{0}}}$ and $g_{\lambda}: V_{\lambda_{0}} \rightarrow \mathscr{B}(K)$ by

$$
g_{\lambda}(x)=\left\{\begin{array}{cc}
\frac{f(x)+c(x)}{\lambda-x} & \text { for } \lambda \neq x, \\
0 & \text { for } \lambda=x .
\end{array}\right.
$$

Note that $g_{\lambda}(x)=0$ on $\bar{V}_{\lambda_{0}}$, so $g_{\lambda}$ is bounded, $\left(\lambda \dot{I}-\dot{M}_{x}\right) \dot{g}_{\lambda}=\dot{f}$.

It follows that $\lambda_{0} \in \varrho_{\dot{M}_{x}}(\dot{f})$; therefore $\mathbb{C} \backslash \bar{D}_{f} \subset \varrho_{\dot{M}_{x}}(\dot{f}) \subset \mathbb{C} \backslash D_{f}$ and $\mathbb{C} \backslash \bar{D}_{f}$ and $\varrho_{\dot{M}_{x}}(\dot{f})$ are open sets. We conclude that $\varrho_{\dot{M}_{x}}(\dot{f})=\mathbb{C} \backslash \bar{D}_{f}$.

We denote $Y_{F}=\{f \in \mathscr{C}(K) \mid \operatorname{Supp} f \subset F\}$ for $F$ closed in $\mathbb{C}$.

7. $Y_{F}$ is analytically invariant for $M_{x}$. Let $f: D \rightarrow \mathscr{B}(K)$ be an analytic function with $\left(\lambda I-M_{\lambda}\right) f_{\lambda} \in Y_{F}$ for every $\lambda \in D$. Therefore $\left(\lambda I-M_{x}\right) f_{\lambda}=g_{\lambda}$ with $\lambda \rightarrow g_{\lambda}$ analytic and $g_{\lambda} \in Y_{F}$ for every $\lambda \in D$. We can show as in the case of $\mathscr{C}(K)$ that $f_{\lambda}$ is continuous. Now, we know that $(\lambda-x) f_{\lambda}(x)=g_{\lambda}(x)$ for every $x \in K$; it follows that $\operatorname{Supp} f_{\lambda}=\operatorname{Supp} g_{\lambda} \subset F$. Hence $f_{\lambda} \in Y_{F}$.

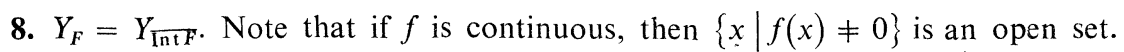
Hence, if we require $\operatorname{Supp} f \subset F, F$ cannot have the void interior $(F \supset\{x \mid f(x) \neq 0\})$.

Now the following equivalences are obvious:

$$
\begin{gathered}
\operatorname{Supp} f \subset F \Leftrightarrow\{x \mid f(x) \neq 0\} \subset F \Leftrightarrow\{x \mid f(x) \neq 0\} \subset \operatorname{Int} F \Leftrightarrow \\
\Leftrightarrow \operatorname{Supp} f \subset \operatorname{Int} F,
\end{gathered}
$$

and the statement follows.

9. $\sigma\left(M_{x} \mid Y_{F}\right)=\overline{\operatorname{Int} F}$. a) We prove that Int $F \subset \sigma\left(M_{x} \mid Y_{F}\right)$, whence $\overline{\operatorname{Int} F} \subset$ $\subset \sigma\left(M_{x} \mid Y_{F}\right)$.

Let $\lambda_{0} \in \varrho\left(M_{x} \mid Y_{F}\right)$; it follows that $\left(\lambda_{0} I-M_{x} \mid Y_{F}\right)$ is surjective, thus for every $g \in Y_{F}$, in particular $g$ with Supp $g \subset F$, there is $f \in Y_{F}$ with $\left(\lambda_{0} I-M_{x}\right) f=g$, or $\left(\lambda_{0}-x\right) f(x)=g(x)$ for every $x \in K$. Note that Supp $f=\operatorname{Supp} g=F$, so that $\{x \mid f(x) \neq 0\} \subset \operatorname{Int} F$. 
From $f(x)=g(x) /(\lambda-x)$ it follows that the problem of existence of $f(x)$ is not trivial only at the points at which $g(x) \neq 0$, that is, at those at which $f(x) \neq 0 . \lambda_{0}$ must be different from those points, therefore $\lambda_{0} \in \mathcal{C}$ Int $F$.

b) We prove that $\sigma\left(M_{x} \mid Y_{F}\right) \subset F$. This, in virtue of 8 , implies that $\sigma\left(M_{x} \mid Y_{F}\right) \subset$ c Int $F$.

Let $\lambda_{0} \in C F$. We prove that $\left(\lambda_{0} I-M_{x} \mid Y_{F}\right)$ is invertible.

Indeed, let $f \in Y_{F}$ with $\left(\lambda_{0} I-M_{x}\right) f=0$, that is $\left(\lambda_{0}-x\right) f(x)=0$ for every $x \in K$. Let $x$ be such that $f(x) \neq 0$, that is $x \in \operatorname{Int} \operatorname{Supp} f \subset F$. Because $\lambda_{0} \in C F$, we also have $\lambda_{0}-x \neq 0$, a contradiction. Hence $f \equiv 0$.

Let now $g \in Y_{F}$. Define

$$
f(x)=\left\{\begin{array}{ccc}
\frac{g(x)}{\lambda_{0}-x} & \text { for } & x \neq \lambda_{0}, \\
0 & \text { for } & x=\lambda_{0} .
\end{array}\right.
$$

Therefore $\left(\lambda_{0} I-M_{x}\right) f=g$ and $\operatorname{Supp} f=\operatorname{Supp} g \subset F, \lambda_{0} \notin F$, hence $f \in Y_{F}$.

Denote $E=X \backslash$ Int $F$ and $\operatorname{Supp}_{E} f=\{\overline{x \in E} \mid f(x) \neq 0\}$.

10. For every $f \in \mathscr{B}(K) \backslash Y_{F}, \sigma_{\dot{M}_{x} F}\left(\dot{f}^{F}\right)=\bar{D}_{f} \cup \operatorname{Supp}_{E} f$. We prove that

$$
\varrho_{M_{x} F}\left(\dot{f}^{F}\right)=(\mathbb{C} \backslash K) \cup\left[K \backslash\left(\bar{D}_{f} \cup \operatorname{Supp}_{E} f\right)\right] .
$$

a) Analogously to 6 we show that

$$
(\mathbb{C} \backslash K) \cup\left[K \backslash\left(\bar{D}_{f} \cup \operatorname{Supp}_{E} f\right)\right] \subset \varrho_{\dot{M}_{x} F}\left(f^{F}\right) .
$$

We obtain $V_{\lambda_{0}}$ from the continuity of $f$; on the other hand, $\lambda_{0} \notin \operatorname{Supp}_{E} f$, therefore there exists $W_{\lambda_{0}}$ such that $f(x)=0$ for every $x \in K \backslash \operatorname{Int} F, x \in W_{\lambda_{0}}$.

Now we define a continuous mapping $c: K \rightarrow \mathbb{C}$, such that $\left.c\right|_{\overline{W_{\lambda_{0}} \cap V_{\lambda_{0}}}}=-\left.f\right|_{\overline{W_{\lambda_{0} \cap V} V_{\lambda_{0}}}}$ and $c=0$ outside $W_{\lambda_{0}} \cap V_{\lambda_{0}}$.

Let $x \in K \backslash$ Int $F$; if $x \in W_{\lambda_{0}} \cap V_{\lambda_{0}}$, then $f(x)=0$, so that $c(x)=0$ as well. If $x \notin W_{\lambda_{0}} \cap V_{\lambda_{0}}$ then $c=0$ by definition. Therefore $x \in K \backslash \operatorname{Int} F$ implies $c(x)=0$, so that $\{x \mid c(x) \neq 0\} \subset$ Int $F \subset F$, hence Supp $c \subset F$.

It follows that $c \in Y_{F}$, which was required.

b) Let now $\lambda_{0} \in \varrho_{M_{x} F}\left(f^{F}\right)$. We have to show that $\lambda_{0} \in C K U\left(K \cap C \bar{D}_{f} \cap \mathcal{C} \operatorname{Supp}_{E} f\right)$.

If $\lambda_{0} \in C K$, we have nothing to prove.

If $\lambda_{0} \in K$, we use the hypothesis: there is an open set $\omega \rightarrow \lambda_{0}$ and there is an analytic function $\dot{h}^{F}: \omega \rightarrow \overline{\mathscr{B}(K)^{F}}$ with $\left(\lambda \dot{I}^{F}-\dot{M}_{x}^{F}\right) \dot{h}_{\lambda}^{F}=\dot{f}^{F}$ on $\omega$, that is

$$
(\lambda-x) h_{\lambda}(x)-f(x)=g_{\lambda}(x)
$$

with $\lambda \rightarrow g_{\lambda}$ analytic, $g_{\lambda} \in Y_{F}$ for every $\lambda \in \omega$.

We show as in 6) that $\lambda_{0} \in K \backslash D_{f}$.It remains to prove that $\lambda_{0} \in K \backslash \operatorname{Supp}_{E} f=$ $=K \cap C \overline{\{x \in K \backslash \operatorname{Int} F \mid f(x) \neq 0\}}=K \cap \operatorname{Int}(\{x \mid f(x)=0\} \cup C K \cup \operatorname{Int} F)$. 
We know that $\lambda_{0} \in K$ and that there is an open set $\omega, \omega \rightarrow \lambda_{0}$. Let us prove that $\omega \subset\{x \mid f(x)=0\} \cup C K \cup$ Int $F$.

Let $\lambda \in \omega$; if $\lambda \in \operatorname{Int} F$, the proof is finished. If $\lambda \in \mathcal{C}$ Int $F=\overrightarrow{C F}$ and $\lambda \in \mathcal{C} K$, the proof is also finished. Let $\lambda \in K, \lambda \in \overline{C F}$.

We know that Supp $g_{\lambda} \subset F$, so that $g_{\lambda}$ being continuous, $\left\{x \mid g_{\lambda}(x) \neq 0\right\} \subset \operatorname{Int} F$, hence $\overline{C F} \subset\left\{x \mid g_{\lambda}(x)=0\right\}$. Hence $g_{\lambda}(\lambda)=0$ and, taking $x=\lambda$ in (1), we obtain $f(\lambda)=0$, what we needed.

The rest of the proof proceeds as in 6).

\section{References}

[1] Apostol, C.: Spectral Decompositions and Functional Calculus, Revue Roum. Math. Pures et Appl., XIII, 10 (1968), p. 1481-1528.

[2] Apostol, C.: Remarks on the Perturbation and a Topology for Operators, J. of Functional Analysis, II, 4 (1968), p. 395-408.

[3] Bacalu, I.: Descompuneri spectrale reziduale, I, Studii și cerc. mat., 32, 5 (1980), p. 467-504.

[4] Bourbaki, N.: Topologie générale, Ch. IX, Hermann, Paris, 1961.

[5] Colojoară, I., Foiaș, C.: Theory of Generalized Spectral Operators, Gordon and Breach, New York, 1968.

[6] Erdelyi, I., Lange, R.: Spectral Decompositions on Banach Spaces, Lecture Notes in Math. nr. 623, Springer, 1977.

[7] Radjabalipour, M.: Decomposable operators, Bull. Iranian Math. Soc. 9 (1978), p. 1-49.

[8] Vasilescu, F. H.: Spectral Distance of two Operators, Revue Roum. Math. Pures et Appl., XII, 5 (1967), p. 733-736.

[9] Vasilescu, F. H.: On an Asymptotic Behaviour of Operators, Revue Roum. Math. Pures et Appl., XII, 3 (1967), p. 353-358.

[10] Vasilescu, F. H.: Residually Decomposable Operators in Banach Spaces, Tôhoku Math. J., 21,4 (1969), p. $509-522$.

[11] Vasilescu, F. H.: Calcul funcțional analitic multidimensional, Edit. Academiei RSR, București, 1979.

[12] Vasilescu, F. H.: Operatori rezidual decompozabili în spații Fréchet, Studii și cerc. matem., 21,8 (1969), p. $1181-1248$.

Author's address: Institute of Mathematics Bucharest, Romania. 